SERGIO QUILICI BELCZAK

\title{
Tratamento endovascular de trauma arterial periférico com o uso de stents revestidos: estudo experimental em porcos
}

Tese apresentada à Faculdade de Medicina da Universidade de São Paulo para obtenção do título de Doutor em Ciências

Programa de: Clínica Cirúrgica

Orientador: Prof. Dr. Erasmo Simão da Silva

São Paulo

2011 
Dados Internacionais de Catalogação na Publicação (CIP)

Preparada pela Biblioteca da

Faculdade de Medicina da Universidade de São Paulo

Creprodução autorizada pelo autor

\section{Belczak, Sergio Quilici}

Tratamento endovascular de trauma arterial periférico com o uso de stents revestidos : estudo experimental em porcos / Sergio Quilici Belczak. -- São Paulo, 2011.

Tese(doutorado)--Faculdade de Medicina da Universidade de São Paulo. Programa de Clínica Cirúrgica.

Orientador: Erasmo Simão da Silva.

Descritores: 1.Procedimentos endovasculares 2.Experimentação animal 3.Stents 4.Traumatismos das artérias carótidas

USP/FM/DBD-216/11 


\section{Dedicatória}

A meu amado pai, o Cirurgião Vascular João Belczak Neto.

A lembrança de seu caráter e dignidade incomparáveis, será presença constante a nortear as nossas vidas. 


\section{Agradecimentos}

Sou grato a Deus, autor de todo o bem, por me ter permitido abraçar esta nobre profissão, e aprender o meu ofício nesta distinguida e reconhecida Instituição da Universidade de São Paulo (USP). Alcançar agora o objetivo maior de obter um Doutorado em Medicina é motivo de grande realização para mim.

Agradeço aos dignos professores e colegas do Serviço de Cirurgia Vascular do Hospital das Clínicas de São Paulo, por todo desvelo e competência profissional que sempre me demonstraram e pelos inestimáveis ensinamentos que paciensiosamente me passaram durante os anos de convivência.

De modo especial, reitero minha gratidão aos Professores Doutores Pedro Puech-Leão e Nelson de Luccia chefes do Serviço e líderes de todos nós, pelas excelentes oportunidades concedidas.

Sou e serei sempre muito grato pela orientação abalizada de nossa tese e pela grande amizade do Professor Doutor Erasmo Simão. O senhor tem me provado o quanto de dignidade um profissional sério pode viver e ensinar aos demais pelo próprio exemplo de sua trajetória de vida.

Ao Professor Doutor Ricardo Aun meu efusivo e eterno muito obrigado por ter sido um mestre incansável, modelo de capacidade profissional. O senhor sempre se mostrou incondicionalmente disposto a me ajudar em todas as minhas necessidades, desde as básicas até às mais sofisticadas.

Meu muito obrigado ao Professor Doutor Luiz Francisco Poli de Figueiredo, Professor Titular da Técnica Cirúrgica. Sua visão científica e, especialmente sua generosidade ao me brindar esta chance de crescimento, muito me sensibilizou. Confirmo mais uma vez a grande admiração que todos compartilhamos pela sua pessoa como ser humano e profissional.

Sou grato pela enorme ajuda concedida pelo Professor Doutor Ivan Bernaduce Casela na discussão das ultrassonografias e análises estatísticas. A solidez de seu conhecimento sobre os referidos temas é motivo de grande respeito por todos nós. 
Agradeço também ao veterinário Professor Doutor Alessandro Belon por ter com tanta amabilidade e competência anestesiado e cuidado dos animais utilizados neste trabalho.

Meu muitíssimo obrigado ao Doutor Vitor C. Gornati pelo valioso auxílio e participação em todas as cirurgias.

Sou profundamente grato ao nosso amigo pessoal o Doutor Igor Rafael Sincos. Sem sua amizade de tantos anos, seu apoio e participação em todos os procedimentos, além da realização dos exames de Eco- Doppler em todos os animais, certamente teria sido muito mais difícil alcançar este marco. Você amigo, tem sido mais que um verdadeiro irmão. Agradeço também o apoio de sempre de meus amigos Luciano Antonio Nassar Pellegrino e Fabrício Ribeiro, grandes amigos e grandes médicos.

Minha eterna e imensa gratidão aos Professores Doutores Paulo Kauffmann e Baptista Muraco. Os senhores são e representam, para nosso orgulho, a glória de uma geração de notáveis Cirurgiões Vasculares que certamente sedimentaram e caracterizaram a grandiosidade da Angiologia e Cirurgia Vascular deste País. Influenciaram positivamente gerações de médicos brilhantes e vocacionados. Herdei de meu pai, ex-aluno de vocês, o respeito, a admiração e o carinho que ele mesmo lhes dedicava.

Confirmo também minha gratidão a todos os trabalhadores do escritório que cuida da Pós-Graduação da Faculdade de Medicina da Universidade de São Paulo. Vocês foram extremamente gentis sempre que precisei de algum esclarecimento e/ou favor.

Agradeço, imerso em pesar, ao meu querido e falecido pai, o saudoso Doutor João Belczak Neto. Amante da Cirurgia Vascular, grande médico, extremamente capaz e caridoso, foi amigo de seus pacientes a quem muito respeitava. Sei que estaria satisfeito e mais que orgulhoso por me ver vencer mais esta etapa.

Meu muito obrigada à minha mãe a Professora Doutora Cleusa Ema Quilici Belczak, médica dedicada e que procurou heroicamente conciliar suas atividades e me estimular na busca da realização de meus sonhos. A certeza de seu amor e confiança, alicerçou cada passo de minha carreira. Seu esforço, obstinação e entusiasmo pelo trabalho e pela especialidade, só fez brotar em mim o desejo de imitá-la.

Ao meu avô Sergio Quilici (hoje, ausente no físico) e à minha amada e sábia avó Maria Antonieta Russo Quilici, meu sincero e profundo agradecimento pelo amor e suporte ao me acolher em sua casa durante minha especialização em São Paulo sem dúvida este foi um dos itens importantes para sedimentar o direito de cursar este Doutorado. Vocês 
jamais disfarçaram o entusiasmo que sentiam pela carreira médica de toda a família Belczak.

Agradeço sensibilizado pelo constante carinho recebido por parte de meu amado irmão o Doutor Rafael Belczak. O indisfarçável orgulho com que se refere à minha pessoa tem sido motivo de estímulo para as minha conquistas e progresso.

A minha querida tia Cleide Ema Quilici que com sua força interior e dinamismo, sempre colaborou nos momentos mais difíceis. Sem a sua dedicação e eficiência nos interesses da família, eu não estaria livre para me dedicar aos nossos trabalhos relacionados à profissão médica.

Finalmente meu obrigado à Doutora Emanuele Lima Villela pela constante paciência e tolerância nos momentos em que necessitei permanecer afastado para dar continuidade aos estudos desta tese. 
Esta tese está de acordo com normas vigentes no momento de sua publicação, conforme seguem:

- Universidade de São Paulo. Faculdade de Medicina. Faculdade de Medicina. Serviço de Biblioteca e Documentação. Guia de apresentação de dissertações, teses e monografias. [Elaborado por Anneliese Carneiro da Cunha, Maria Julia de A. L. Freddi, Maria F. Crestana, Marinalva Souza Aragão, Suely Campos Cardoso, Valéria Vilhena]. 2. ed. São Paulo: Serviço de Biblioteca e Documentação; 2011.

- International Committee of Medical Journals Editors (Vancouver), adaptado para citações e referências bibliográficas.

- List of Journals Indexed in Index Medicus, para abreviatura dos títulos dos periódicos.

- Bechara E. Moderna gramática portuguesa. 37. ed. Rio de Janeiro: Lucerna; 2000.

- Bechara E. Novo acordo ortográfico da língua portuguesa. Rio de Janeiro: Nova Fronteira; 2008.

- Stedman: Dicionário médico. [Traduzido sob a coordenação de Sérgio Augusto Teixeira]. 23. ed. Rio de Janeiro: Guanabara Koogan; 1997. [Volumes 1 e 2]. 


\section{Sumário}

Lista de Abreviaturas, símbolos e siglas

Lista de tabelas

Lista de figuras

Resumo

Summary

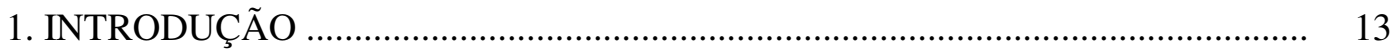

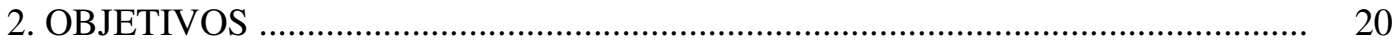

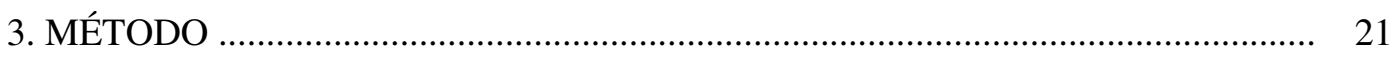

3.1 Procedimentos anestésicos e perioperatórios ........................................................ 24

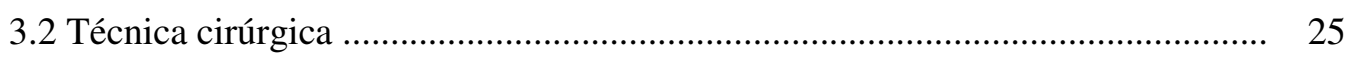

3.3 Avaliação da viabilidade do procedimento ...................................................... 29

3.4 Análise estatística ...................................................................................... 31

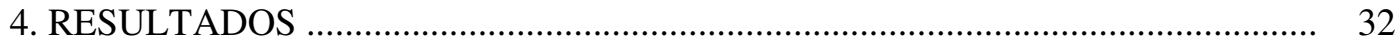

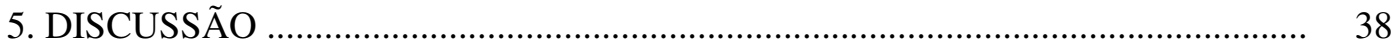

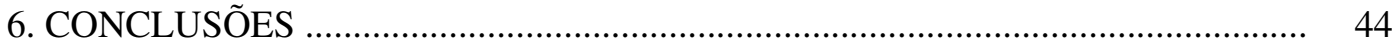

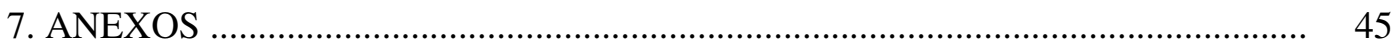

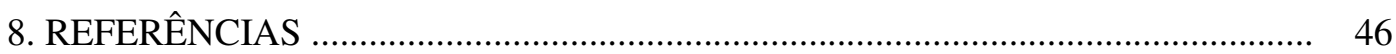




\section{Lista de tabelas}

Tabela 1 Valores do peso (em quilogramas) dos porcos utilizados no estudo e do peso médio do grupo-controle e dos grupos experimentais .

Tabela 2 Valores do tempo operatório total (em minutos) utilizado em cada porco e da média do grupo-controle e dos grupos experimentais

Tabela 3 Valores do tempo operatório pós-trauma controlado (em minutos) utilizado em cada porco e da média do grupo-controle e dos grupos experimentais

Tabela 4 Evidências das imagens arteriográficas antes e depois da liberação de stents revestidos em trauma controlado de artéria carótida comum esquerda

Tabela 5 Valores médios e desvios-padrão dos parâmetros vitais monitorados ao longo do estudo

Tabela 6 Parâmetros ultrassonográficos em 14 procedimentos endovasculares bem sucedidos após produção de trauma perfurante controlado

Tabela 7 Parâmetros ultrassonográficos (média \pm desvio-padrão) avaliados antes e após a colocação de stents revestidos na artéria carótida comum esquerda após a produção de trauma perfurante controlado no conjunto de 14 procedimentos bem sucedidos

Tabela 8 Parâmetros ultrassonográficos (média \pm desvio-padrão) avaliados antes e após a colocação de stents revestidos na artéria carótida comum esquerda após a produção de trauma perfurante controlado no grupocontrole e nos grupos experimentais 


\section{Lista de figuras}

Figura 1 Exposição da artéria carótida comum esquerda ……............................................. 26

Figura 2 Secção controlada da parede do vaso (extensão circunferencial de 33\%) ................. 27

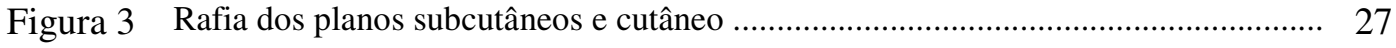

Figura 4 Lesão evidenciada em imagem arteriográfica ....................................................... 28

Figura 5 Imagem arteriográfica do momento em que o fio guia transpassa o local da lesão ... 29

Figura 6 Posicionamento adequado do stent revestido liberado com sucesso ………............. 30

Figura 7 Perviedade arterial e bom fluxo sanguíneo evidenciados em imagem arteriográfica de controle

Figura 8 Imagens arteriográficas pré e pós-tratamento endovascular evidenciando procedimentos com sucesso nos quatro grupos.

Figura 9 Imagem de necrópsia evidenciando os cotos arteriais conectados pelo stent revestido.

Figura10 Anatomia vascular suína evidenciada em reconstrução de angiotomografia computadorizada. 


\section{Lista de Abreviaturas, Símbolos e Siglas}

$\begin{array}{ll}\mathrm{cm} & \text { centímetro(s) } \\ \text { Dr. } & \text { doutor } \\ \text { DAOP } & \text { doença arterial obstrutiva periférica } \\ \mathrm{F} & \text { frenchs } \\ \mathrm{Kg} & \text { quilograma(s) } \\ \mathrm{mg} & \text { miligrama(s) } \\ \mathrm{mm} & \text { milímetros } \\ \mathrm{NaCl} & \text { cloreto de sódio } \\ \text { p. } & \text { páginas } \\ \text { PET } & \text { tereftalato de polietileno } \\ \text { Prof. } & \text { professor } \\ \text { PTFE } & \text { politetrafluoretileno } \\ \mathrm{S} & \text { segundo(s) } \\ \mathrm{U} & \text { unidades }\end{array}$




\section{Resumo}

Belczak SQ. Tratamento endovascular de trauma arterial periférico com o uso de stents revestidos: Estudo experimental em porcos [tese]. São Paulo: Faculdade de Medicina, Universidade de São Paulo; 2011. 50p.

Introdução: Os traumas arteriais e venosos são responsáveis por expressiva morbimortalidade, e, em determinados territórios, a técnica de restauração aberta acrescenta riscos elevados ao paciente, que podem ser minimizados com o uso de técnicas endovasculares. Objetivos: O objetivo deste estudo foi criar um modelo experimental de trauma vascular periférico penetrante em que se avalia a viabilidade do reparo endovascular em lesões da parede arterial com diferentes extensões cincunferenciais. Método: Vinte porcos brancos machos foram divididos em quatro grupos, de acordo com a extensão circunferencial do trauma arterial: sem lesão arterial (Grupo 1); lesão arterial com extensão circunferencial <50\% (Grupo 2); lesão arterial com extensão circunferencial >50\%, variando entre 50-80\% (Grupo 3); e secção completa (Grupo 4). A artéria carótida comum esquerda foi dissecada com controle arterial proximal e distal, procedimento que se seguiu de secção controlada da parede arterial, fechamento dos planos e compressão manual por dez minutos, seguida de tratamento endovascular com introdução de stent revestido Viabahn ${ }^{\mathrm{TM}}(5 \mathrm{~mm} \times 50 \mathrm{~mm})$ por via de acesso femoral. Resultados: A viabilidade e a reprodutibilidade do modelo experimental proposto foram confirmadas pelo sucesso no tratamento de todos os animais sem trauma e nos animais com lesões $<50 \%$. Sucesso da técnica endovascular também foi observado em quatro dos cinco animais com lesões $>50 \%$ e, em um animal com secção completa. Variáveis como a duração do procedimento, parâmetros ultrassonográficos e arteriográficos, e flutuação dos sinais vitais foram devidamente monitoradas. Conclusões: O reparo endovascular do trauma arterial periférico em animais de experimentação mostrou-se factível com limitação dependendo da extensão circunferencial da lesão. Este modelo experimental, envolvendo técnicas endovasculares, indicou etapas importantes a serem consideradas em outros estudos nestes animais e com a utilização destes materiais.

Descritores: Procedimentos endovasculares, Experimentação animal, Stents, Traumatismo das artérias carótidas 


\section{Abstract}

Belczak SQ. Endovascular treatment of peripheral arterial injury with covered stents: An experimental study in pigs [thesis]. São Paulo: Faculdade de Medicina, Universidade de São Paulo; 2011. 50p.

Background: Additional surgical trauma often increases the risk of major morbidity and mortality associated with vascular injury, and endovascular repair could have many advantages in such situations. Objectives: The aim of this study was to create an experimental animal model of penetrating peripheral artery injury and to evaluate the feasibility of endovascular repair in different degrees of circumferential injury. Methods: Twenty white male domestic pigs were divided into four groups according to the circumferential extent of arterial injury: no injury; circumferential injury extent $<50 \%$ or $>50 \%$, ranging between 50 $80 \%$, and complete sectioning. Left common carotid artery was dissected with proximal and distal artery control followed by controlled section of the arterial wall. Local manual compression was applied for 10 minutes followed by endovascular treatment with a 5 x $50 \mathrm{~mm}$ Viabahn ${ }^{\mathrm{TM}}$ covered stent using the femoral approach. Results: The feasibility and reproducibility of the proposed experimental model was confirmed by the successful treatment of all animals with no injury and with injuries with a circumferential extent $<50 \%$. Success was also achieved in four of the 5 animals in the group with injuries of circumferential extent $>$ $50 \%$, and in one pig in the complete section group. Additional variables were monitored, such as duration of procedure, ultrasound and arteriography parameters and fluctuation of vital signs. Conclusions: Endovascular repair of arterial injury is possible depending on circumferential extension of arterial lesion. This experimental model, involving endovascular techniques, shows important steps to consider in further studies in these animals and use of these materials.

Descriptors: Endovascular procedures, Animal experimentation, Stents, Carotid artery injuries 


\section{Introdução}

Traumas de diferentes mecanismos foram considerados a primeira causa de morte em indivíduos com até 44 anos, segundo estudos bem conduzidos, nas décadas de 80 e $90^{1}$. Também são responsáveis pela terceira causa de morte em todo o mundo, independentemente da fase faixa etária ${ }^{2}$.

Lesões vasculares decorrentes desses traumas afetam $3 \%$ da população civil ${ }^{2}$. O acometimento das extremidades superiores e inferiores atingem aproximadamente $70 \%$ desses pacientes. O território carotídeo é responsável por $5 \%$ a $10 \%$ desse tipo de lesão. Em $75 \%$ dos casos, essas lesões ocorrem na artéria carótida comum, usualmente por traumas penetrantes ${ }^{3}$, e em cerca de $20 \%$, na artéria carótida interna, mais frequentemente por traumas contusos ${ }^{4}$.

As consequências imediatas das lesões vasculares periféricas dependem do mecanismo de trauma. Avulsão de grandes vasos, lesões da íntima e dissecções da parede arterial que resultam em trombose são geralmente causadas pelo impacto direto por trauma fechado ${ }^{5}$. Por sua vez, laceração da camada adventícia da parede arterial, bem como secções parciais ou secção completa do vaso resultantes de traumas penetrantes, podem evoluir com alto risco de hemorragia exsanguinante ${ }^{6}$. Tanto o trauma contuso quanto o penetrante podem resultar na formação de pseudoaneurisma ${ }^{7}$. Os pseudoaneurismas pós-traumáticos podem evoluir com trombose, embolização e rotura. As lesões vasculares, portanto, estão associadas com altas taxas de morbidade e mortalidade ${ }^{8}$. 
A vantagem do tratamento endovascular é de promover a exclusão da lesão vascular ou trombose do saco do pseudo-aneurisma com restituição do fluxo sanguíneo normal, via um sítio remoto de acesso e sem inconvenientes de ação cirúrgica direta na área traumatizada ${ }^{9,10}$.

A exploração aberta da lesão seguida do adequado reparo cirúrgico é o tratamento usualmente preconizado em pacientes politraumatizados com instabilidade hemodinâmica e com sinais diretos de trauma vascular periférico como hemorragia e hematoma em progressão ${ }^{2,11}$.

Todavia, em alguns casos, para pacientes com estabilidade hemodinâmica, trauma cirúrgico pode aumentar os riscos para pacientes e, nestes, o tratamento endovascular tem mostrado resultados benéficos ${ }^{11,12}$.

Técnicas endovasculares para lesões em diferentes veias e artérias decorrentes de traumas têm sido descritas com sucesso. Em casos de fratura pélvica decorrente de trauma contuso, por exemplo, técnicas de embolização já constituem indicação terapêutica de primeira escolha ${ }^{13}$.

Vinces e Sperling ${ }^{14}$ relataram o primeiro caso de lesões combinadas, envolvendo pseudoaneurisma e fístula arteriovenosa da artéria subclávia direita, decorrentes de trauma penetrante por projétil de arma de fogo na região clavicular, no qual o uso de técnica endovascular se mostrou alternativa eficaz para esse paciente hemodinamicamente estável. Além do sucesso técnico da intervenção, esses autores relataram ainda outras vantagens da técnica endovascular, como a relação custo-benefício do tratamento, menor desconforto para o paciente, redução da perda sanguínea, do tempo operatório, e do risco das manobras difíceis para a exposição do vaso lesado.

Xenos et al. ${ }^{15}$ trataram sete casos de lesões traumáticas (contusas e perfurantes) 
das artérias subclávia e axilar. Observaram a viabilidade do procedimento em pacientes adequadamente selecionados, nos quais o tempo cirúrgico e a perda sanguínea foram significativamente menores do que de outros pacientes da mesma série que foram tratados com cirurgia aberta. Segundo os autores, a abordagem endovascular em lesões de artérias subclávia e axilar reduz o risco de lesar estruturas adjacentes importantes (como o nervo vago, o nervo laríngeo recorrente, o nervo frênico e a veia inominada), uma vez que elimina a necessidade aguda de dissecção cirúrgica.

O segmento arterial femoropoplíteo é o mais acometido por lesões vasculares traumáticas. Parodi e cols já evidenciaram os benefícios da utilização de stents revestidos na artéria femoral comum e superficial em pacientes vítimas de lesões penetrantes nestes vasos ${ }^{16}$. Há relatos de tratamento endovascular bem-sucedido na artéria poplítea, entretanto, stents geralmente são inadequados nesta localização em virtude da dificuldade destes dispositivos se acomodarem na região de flexão do joelho ${ }^{17}$.

Spirito et al. ${ }^{18}$ relataram o caso de paciente idoso que, após trauma contuso nãopenetrante, desenvolveu um pseudo-aneurisma da artéria tibial anterior associado com fístula arteriovenosa, e o sucesso do tratamento endovascular foi confirmado um ano depois do procedimento.

Em relação ao trauma no território carotídeo, a taxa geral de mortalidade alcança 66\%. As taxas de mortalidade e ocorrência de infarto cerebral geralmente são maiores nas lesões da artéria carótida interna do que nas lesões da artéria carótida comum. Du Toit et al. ${ }^{4}$ reportaram mortalidade em $31 \%$ e ocorrência de isquemia cerebral em 23\% dos indivíduos com lesões penetrantes da artéria carótida interna.

As técnicas endovasculares oferecem benefícios potenciais nos casos de lesões da artéria carótida interna distal ${ }^{19-21}$, local em que a exploração direta exige dissecção 
extensa ou subluxação da mandíbula para a boa exposição da artéria, além de estar associada com taxa de $9 \%$ de isquemia cerebral perioperatória ${ }^{22}$.

Em estudo retrospectivo em que se compararam as opções terapêuticas para lesões contusas da artéria carótida, Li et al. ${ }^{23}$ não encontraram vantagens no uso de abordagens endovasculares. Por outro lado, ampla revisão de literatura publicada por Du Bose et al. ${ }^{24}$ evidenciou que o tratamento de lesões carotídeas tanto perfurantes quanto contusas resultou em taxa de perviedade de quase $80 \%$, sem mortalidade relacionada ao procedimento, embora déficits neurológicos novos tenham ocorrido em 3,5\% dos casos após o posicionamento do stent.

O fato é que a tomada de decisão para a indicação de tratamento endovascular para o tratamento de lesões carotídeas traumáticas exige, nos dias de hoje, avaliação cuidadosa do paciente quanto à estabilidade hemodinâmica, ao quadro neurológico, à presença de hemorragia ativa e ao tipo de trauma. Essa decisão ainda é controversa e estritamente individualizada ${ }^{25}$.

Portanto, nas lesões traumáticas de vasos de diferentes diâmetros o tratamento pela técnica endovascular já foi realizado de modo empírico sem uma real constatação dos seus efeitos biológicos e fisiológicos, como também com informações limitadas quanto a sua durabilidade.

No tratamento da doença arterial obstrutiva periférica (DAOP), o número de intervenções endovasculares vem crescendo cada vez mais, e o rápido avanço nas técnicas e dispositivos pertinentes está permitindo que os resultados dessas intervenções se aproximem cada vez mais dos resultados obtidos com as cirurgias vasculares tradicionais $^{26}$. Para esses casos, de lesões degenerativas não traumáticas, embora a angioplastia com balão ofereça bons resultados imediatos, a implantação de stents tem 
sido uma opção para otimizar o sucesso do procedimento endovascular e ampliar a possibilidade desse tratamento a maior número de pacientes com DAOP. A experiência inicial com pacientes traumatizados submetidos a correção endovascular com próteses (stents, stents revestidos) foi uma projeção da grande experiência neste campo (DAOP).

O termo stent (também conhecido como endoprótese) deriva do nome de Charles Thomas Stent, dentista que viveu entre 1807 e 1885, que foi o primeiro profissional a criar um material maleável que pudesse manter enxertos de pele na cavidade oral $^{26}$. Hoje, os stents são tubos metálicos que servem para ser inseridos no lúmen vascular, de modo a manter a abertura do lúmen arterial com o uso de pressão mecânica (tensão radial) ${ }^{27}$.

Esses dispositivos podem ser classificados de acordo com a forma de introdução; o mecanismo de implantação (auto-expansível, balão expansível); o tipo de liga metálica; a composição do metal de que é feito; o desenho estrutural (tubo ou mola); e a geometria, ou seja, o formato dos anéis que o compõem (em rede aberta ou fechada $)^{28}$. Para avaliar cada uma dessas características em diferentes sítios vasculares, uma série de estudos experimentais vem sendo realizada ao longo dos últimos anos ${ }^{29-}$ 35 .

As propriedades ideais dos stents endovasculares incluem alta radio-opacidade para melhor visualização e para implantação mais fácil; força radial adequada que resista remodelamento elástico; sistema de implantação simples e de fácil manuseio; flexibilidade longitudinal que permita navegar por bifurcações, vasos de diferentes calibres e tortuosidades; elasticidade radial que resista compressão externa sem deformação permanente; perfil pequeno com ampla expansão que possa navegar em 
pequenos vasos e estenoses críticas; encolhimento mínino após a sua liberação; mínima indução de hiperplasia; resistência contra trombose e corrosão; durabilidade; e baixo custo ${ }^{26,29,32}$.

O sucesso do implante de um stent também depende do desenvolvimento de trombose mínima e endotelização rápida secundárias ao implante deste dispositivo. Imediatamente após o procedimento, há exposição de elementos da camada subíntima ao fluxo sanguíneo, o que pode resultar em deposição plaquetária e formação trombótica, proliferação de fibroblastos e hiperplasia da camada íntima. O fluxo sanguíneo pode se tornar turbulento no local da dissecção em decorrência da irregularidade da camada íntima, o que pode promover deposição excessiva de plaquetas e fibrina $^{36,37}$. Está bem estabelecido que a heparinização e/ou a utilização de antiagregantes plaquetários previnem a formação de trombose dentro do stent $^{37}$. Com o intuito de minimizar o potencial trombogênico desse dispositivo e de impedir a hiperplasia da camada íntima, diversos estudos vêm sendo realizados focalizando tanto novos materiais para a fabricação dos stents quanto o desenvolvimento de substâncias que os revistam ${ }^{26,28,38-40}$.

O equilíbrio entre a resistência da parede vascular e a força radial do stent é o que vai determinar a fixação do dispositivo. A reação local que se estabelece em decorrência do contato dos metais de que os stents são feitos com a parede vascular pode interferir na sua patência ao longo do tempo. Por essa razão, foram desenvolvidos revestimentos com soluções e películas com baixa trombogenicidade e que reduzam a agregação plaquetária pela inibição da migração de elétrons para os metais ${ }^{40}$.

Os materiais de revestimento estudados experimentalmente incluem politetrafluoretileno $(\mathrm{PTFE})^{41-46}$, poliéster $\left(\text { Dacron }^{\circledR}\right)^{47-51}$, tereftalato de polietileno $(\mathrm{PET})^{47}$, 
poliuretano $^{49}$, ligas de carbono ${ }^{52,53}$, associados com substâncias ou polímeros contendo drogas antitrombóticas e antiproliferativas ${ }^{54-60}$, de forma também a prevenir a hiperplasia da íntima ${ }^{28}$. Diversos estudos clínicos, com casuísticas pequenas e geralmente publicados na forma de metanálises, já atestaram a eficácia de procedimentos endovasculares com stents revestidos no tratamento de lesões vasculares decorrentes de traumas ${ }^{4,12-18,61}$, incluindo lesões na artéria carótida ${ }^{4,22,24,25}$. Como já mencionado, estes estudos e os grupos que os realizaram partiram de experiência prévia com o uso de endopróteses em artérias degeneradas. A questão do comportamento em artérias de indivíduos geralmente jovens (os traumas ocorrem preferencialmente em indivíduos sem doença degenerativa e abaixo dos 40 anos de idade) e qual seria o material ideal são uma incógnita, com necessidade de estudos e modelos de estudos experimentais que possam analisar antecipadamente não apenas a técnica de utilização, mas também a biologia destes materiais em contato com as artérias ${ }^{4}$.

Da mesma forma, considera-se que o tratamento endovascular não é o mais indicado em casos de secção arterial completa ${ }^{16}$, uma vez que parece impossível a cateterização do coto arterial distal a lesão traumática; mas ainda não há evidências que suportem essa assertiva, assim como ainda não existem estudos sobre a eventual associação entre a extensão circunferencial da lesão traumática na parede arterial e a viabilidade do reparo endovascular. 


\section{Objetivos}

Foram objetivos deste estudo:

1. A descrição de um modelo animal que permita avaliar a viabilidade do reparo endovascular com uso de stents revestidos em lesões vasculares periféricas decorrentes de traumas penetrantes.

2. Avaliar a possibilidade de reparo com técnica endovascular, utilizando stents revestidos, em diferentes extensões de lesão circunferencial da parede arterial. 


\section{Método}

Este estudo foi realizado na Disciplina de Técnica Cirúrgica do Departamento de Cirurgia da Faculdade de Medicina da Universidade de São Paulo e no setor de Cirurgia Experimental do Instituto do Coração do Hospital das Clínicas da Faculdade de Medicina da Universidade de São Paulo, entre abril de 2009 e fevereiro de 2010.

O modelo animal objeto do estudo foi desenvolvido com 20 porcos brancos domésticos machos, que pesavam entre $28 \mathrm{~kg}$ e 37,5 $\mathrm{kg}$ (peso médio de 31,3 $\pm 2,5 \mathrm{~kg}$ ). Não houve diferença estatística $(p=0,4253)$ entre os grupos com relação a essa variável, conforme dados apresentados na Tabela 1.

Tabela 1. Valores relativos ao peso (em quilogramas) dos porcos utilizados no estudo e ao peso médio do grupo-controle e dos grupos experimentais.

\begin{tabular}{|c|c|c|c|c|}
\hline \multirow[b]{2}{*}{ Porcos } & \multicolumn{4}{|c|}{ Grupos } \\
\hline & $\begin{array}{c}\text { G1 } \\
\text { (controle) }\end{array}$ & $\begin{array}{c}\text { G2 } \\
(33 \%)\end{array}$ & $\begin{array}{c}\text { G3 } \\
(66 \%)\end{array}$ & $\begin{array}{c}\text { G4 } \\
(110 \%)\end{array}$ \\
\hline 1 & 29,0 & 37,5 & 34,3 & 34,0 \\
\hline 2 & 29,0 & 32,0 & 31,8 & 28,5 \\
\hline 3 & 28,0 & 33,5 & 31,0 & 32,0 \\
\hline 4 & 32,0 & 32,0 & 28,0 & 29,0 \\
\hline 5 & 31,5 & 31,0 & 33,0 & 28,0 \\
\hline Média \pm desvio-padrão & $29,9 \pm 1,7$ & $33,2 \pm 2,6$ & $31,6 \pm 2,4$ & $30,3 \pm 2,6$ \\
\hline
\end{tabular}

Foram rigorosamente adotadas e seguidas as recomendações e normatizações prescritas pelo Decreto 6.899, datado de 15 de julho de 2009, para uso de animais em experimentos científicos e para proteção desses animais.

Para os procedimentos experimentais, foram formados quatro grupos com cinco 
porcos. No primeiro grupo (G1), que serviu de grupo-controle, não foi provocada qualquer lesão controlada da artéria carótida nos animais, e a viabilidade do procedimento proposto foi analisada em modelos de vasos sem lesão traumática.

Nos outros três grupos, foram produzidos traumas controlados da artéria carótida dos animais, a saber:

- No Grupo 2 (G2), a extensão circunferencial do trauma controlado provocado na parede arterial foi de $33 \%(<50 \%)$.

- No Grupo 3 (G3), a extensão circunferencial do trauma controlado provocado na parede arterial foi de $66 \%(>50 \%)$.

- No Grupo 4 (G4), foi produzida secção completa (100\%) na parede da artéria carótida dos animais.

A mensuração da extensão circunferencial da lesão arterial foi subjetiva baseada na opinião de dois pesquisadores. Os animais foram operados em ordem sequencial de acordo com seu respectivo grupo, inicialmente o G1, após o G2 e assim por diante até o termino do estudo.

Arteriografias, com a utilização do equipamento Philips BV Pulsera RX $0930^{\circledR}$, foram realizadas antes e após o procedimento, avaliando a perviedade, presença de espasmo arterial e extravasamento de contraste (Ioxitalamato de meglumina - Telebrix $\left.{ }^{\circledR}\right)$.

Ultrassonografias Doppler, com a utilização do equipamento Sonosite $\operatorname{Titan}^{\circledR}$, foram realizadas antes e após o procedimento. Todos os exames ultrassonográficos foram realizados pelo Doutor Igor Rafael Sincos, um dos pesquisadores envolvidos no estudo. Foram avaliados o diâmetro arterial máximo (em centímetros), a velocidade de pico sistólico (em centímetros por segundo), a velocidade de pico diastólico 
(em centímetros por segundo) e o índice de resistência, que é a subtração da velocidade diastólica máxima da velocidade sistólica máxima dividida pela velocidade sistólica máxima, e é muito utilizado para avaliar alterações de velocidade em regiões de estenose.

A realização do estudo-piloto com quatro porcos, solucionou questões técnicas antes do início dos experimentos. Nesse sentido, a primeira dificuldade a ser solucionada foi a ocorrência de vasoespasmos, que é bastante frequente nos estudos experimentais realizados com porcos, cujos vasos sanguíneos periféricos são suscetíveis a espasmo e a fácil rotura ${ }^{62}$. Essa dificuldade foi resolvida com infiltração local de xilocaína a $2 \%$ e administração de papaverina, imediatamente após a liberação do stent, na dose de $0,7 \mathrm{mg} / \mathrm{kg}$.

Outra dificuldade, também solucionada nos estudos pilotos, foi a formação de trombos no introdutor e no stent imediatamente após sua liberação. Neste animais, é necessário a utilização de doses maiores que a utilizada em humanos ${ }^{37,56,63-65}$. Desta forma, para impedir a formação de trombose intra-stent, foi realizada heparinização sistêmica (140 U/kg) após a colocação do introdutor e local pelo cateter (15 U/kg) logo imediatamente após a liberação do stent.

Nossa escolha pelo stent revestido VIABAHN ${ }^{\circledR}$ foi fundamentada tanto nas características do dispositivo, destacando-se sua flexibilidade, autoexpansibilidade, facilidade de liberação, quanto na disponibilidade desse produto em tamanho adequado $(5 \mathrm{~cm} \times 50 \mathrm{~mm})$ para ser utilizado em artéria carótida com a configuração anatômica dessa artéria em porcos. Todos os stents utilizados neste estudo foram doados pela GORE ${ }^{\circledR}$ Inc.

O stent revestido VIABAHN ${ }^{\circledR}$ é um dispositivo endoluminal flexível, autoex- 
pansível, que consiste em um ePTFE (politetraflureno expandido) com suporte de nitinol ao longo de todo o seu comprimento. Oferece fácil liberação, é flexível longitudinalmente e capaz de se adaptar em anatomias mais tortuosas. A válvula de disposição está amarrada a uma fita de ePTFE . Com uma simples tração na válvula, a endoprótese é solta do cateter. A colocação é completada assim que a fibra é retirada de dentro do cateter. O sistema de distribuição permite uma colocação precisa sob fluoroscopia devido ao desenho que não retrai e marcas radiopacas gravadas no cateter.

Todos os animais foram sacrificados imediatamente após a avaliação do sucesso ou insucesso do procedimento. O sacrifício dos animais seguiu rigorosamente as recomendações do referido Decreto 6.899/2009.

\subsection{PROCEDIMENTOS ANESTÉSICOS E PERIOPERATÓRIOS}

O procedimento anestésico foi realizado sob supervisão do Médico Veterinário Alessandro Rodrigo Belon. Todos os animais foram pré-anestesiados com injeção intramuscular de Quetamina (5,0 mg/kg) e Midazolan $(0,25 \mathrm{mg} / \mathrm{kg})$ misturados na mesma seringa.

Passados 15 minutos da injeção, a veia marginal da orelha foi cateterizada com cateter de calibre 20 ou 22 (BD Insystem, Becton Therapy Systems Inc., EUA), para servir de acesso venoso para a indução anestésica, que foi realizada com $20 \mathrm{mg} / \mathrm{kg}$ de tiopental sódico e administração adicional de volume inicial de solução fisiológica $(\mathrm{NaCl})$ a $0,9 \%$ para compensar a perda relacionada ao jejum de oito horas a que os 
animais foram submetidos antes do início dos procedimentos.

Foram utilizados tubos endotraqueais tamanho 6 (Portex $\left.{ }^{\circledR}\right)$ para a intubação dos animais.

A anestesia foi mantida com halotano com concentração de $50 \%$ de oxigênio no ar inspirado e de $1 \%$ a $2 \%$ de halotano no ar expirado, em circuito circular fechado em aparelho de anestesia inalatória $\left(\right.$ Takaoka $\left.^{\circledR}\right)$. A analgesia foi mantida com fentanil, com dose inicial de $5 \mu \mathrm{g} / \mathrm{kg}$, seguida de infusão intravenosa contínua de 0,4 $\mu \mathrm{g} / \mathrm{kg} / \mathrm{min}$.

No monitoramento das funções vitais, parâmetros fisiológicos de respiração foram mantidos durante ventilação mecânica após a intubação endotraqueal. A freqüência cardíaca, pressão arterial invasiva e pressão venosa central foram avaliadas continuamente até o sacrifício do animal. Durante a cirurgia manteve-se a hidratação dos animais com administração de fluídos.

\subsection{TÉCNICA CIRÚRGICA}

Os traumas carotídeos controlados foram produzidos e corrigidos com os animais posicionados em decúbito dorsal.

A artéria femoral direita foi dissecada para cateterização e monitoramento invasivo da pressão arterial. A veia femoral direita foi dissecada para cateterização e monitoramento invasivo da pressão venosa central. Foi realizado, então, incisão cervical longitudinal, e a artéria carótida comum esquerda exposta mediante dissecção meticulosa (Figura 1) . Foi realizada infiltração nos tecidos peri-carotídeos de xilocaína a $2 \%$. 


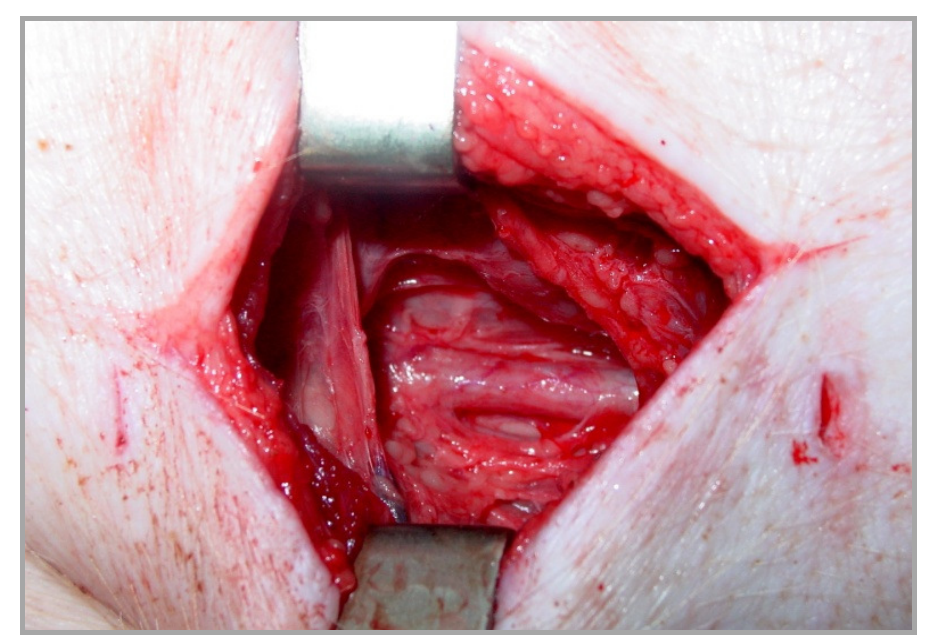

Figura 1. Dissecção da artéria carótida comum esquerda.

Dependendo do grupo de estudo, houve secção controlada da artéria com extensão circunferencial de aproximadamente 33\% (menor que 50\% - Figura 2), 66\% (maior que $50 \%$ ) ou $100 \%$.

Controle proximal e distal da artéria carótida foi realizado por meio de duas pequenas incisões, por contra-abertura, uma distal e uma proximal à cervicotomia, onde foram posicionadas pinças hemostáticas delicadas (aqui denominadas clampes). $\mathrm{Na}$ sequência operatória, a parede arterial foi seccionada (Figura 2), e as camadas musculares profundas, do músculo platisma e cutânea foram suturadas (Figura 3). Esta sutura, em três planos, foi realizada para mimetizar a ação dos tecidos peri-lesionais no trauma perfurante quando não há a incisão de acesso. 

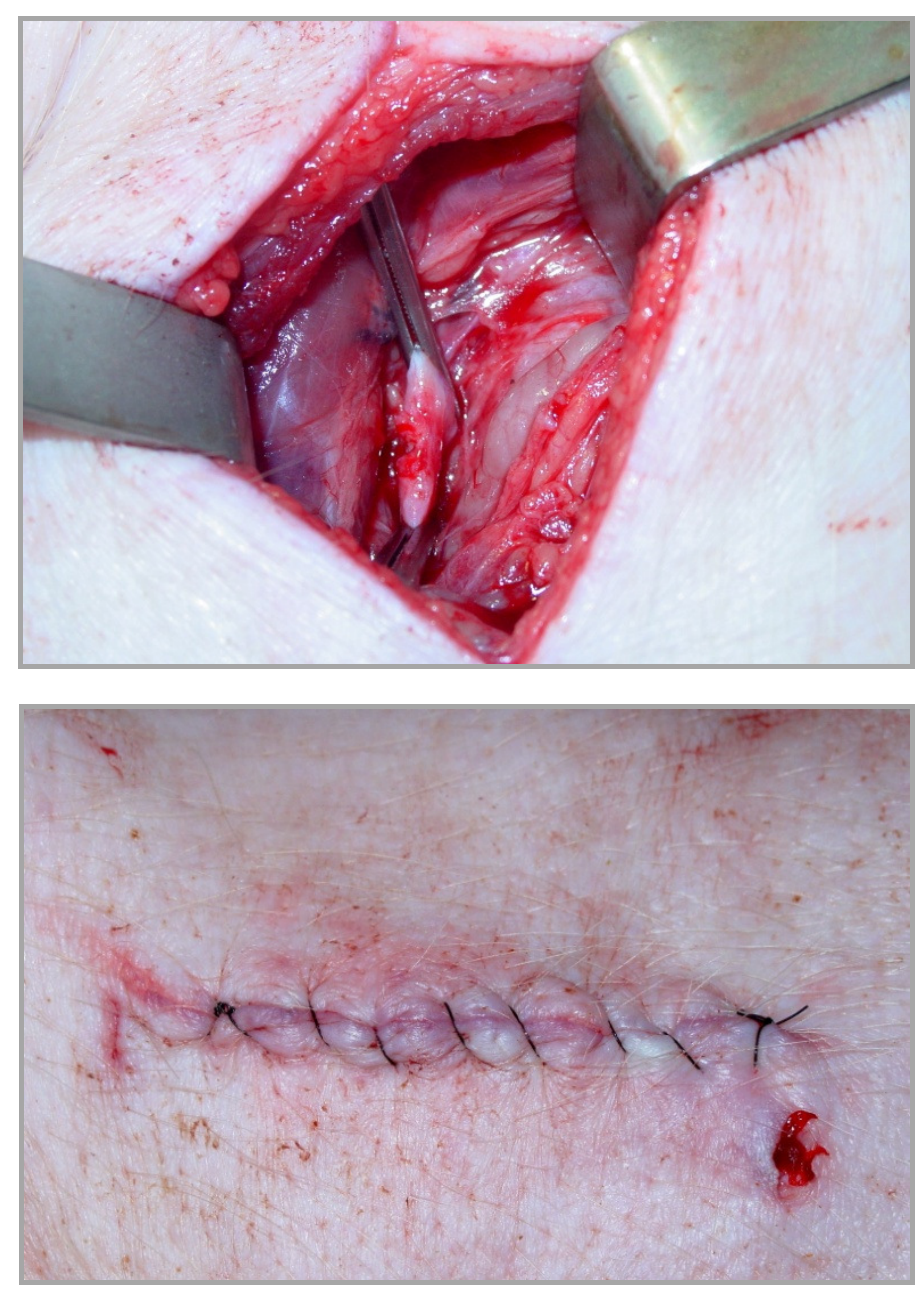

Figura 2. Secção controlada da parede do vaso (extensão circunferencial de $33 \%$ ).

Figura 3. Rafia dos planos subcutâneos e cutâneo. Caracterização do sítio de inserção dos clampes arteriais.

Após a liberação dos clampes vasculares, o local do trauma provocado recebia compressão manual durante reposição volêmica com 20 mg/kg de solução fisiológica $(\mathrm{NaCl})$ a $0,9 \%$ por 10 minutos, antes de ser dado início ao procedimento endovascular. Este período de compressão manual e reposição volêmica caracterizou o período de atendimento pré-hospitalar do paciente vítima de trauma. Todos animais dos grupos com lesão apresentaram neste momento extenso hematoma cervical, entretanto em nenhum caso se caracterizou exteriorização de sangramento após a compressão.

O procedimento endovascular então foi iniciado com a dissecção da artéria femoral comum esquerda, seguida da colocação de introdutor $8 \mathrm{~F}$ pela técnica de Sel- 
dinger e heparinização sistêmica (145 U/kg), para posterior colocação do stent revestido VIABAHN ${ }^{\circledR}$. Nesse momento, foi realizada cateterização da carótida comum esquerda com uso de fio guia hidrofílico de 0,035 polegadas (Aqualiner) e então realizada arteriografia com cateter VERT 5F, de modo a evidenciar o local da lesão (Figura 4). Não se utilizou Roadmap, apenas a fluoroscopia direta para progressão do fio guia distalmente. Quando o fio guia transpassou a lesão (Figura 5), diretamente sobre este, foi liberado o stent revestido VIABAHN ${ }^{\circledR}$, medindo $5 \mathrm{~mm}$ de diâmetro e $5 \mathrm{~cm}$ de comprimento. Realizou-se então, administração via cateter de papaverina na dose de $0,7 \mathrm{mg} / \mathrm{kg}$ e de heparina na dose de $15 \mathrm{U} / \mathrm{kg}$. Não se fez necessário qualquer tipo de balonamento para acomodação do stent.

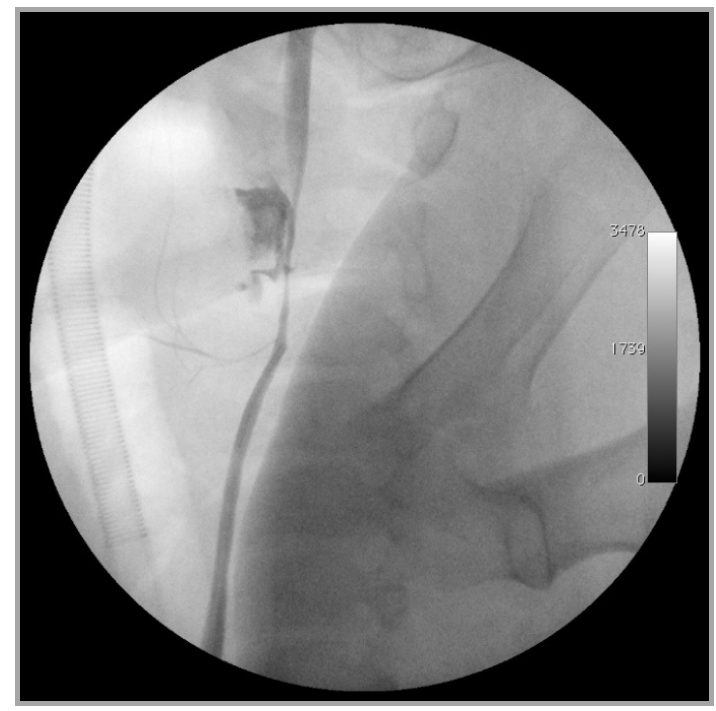

Figura 4. Lesão evidenciada em imagem arteriográfica. 


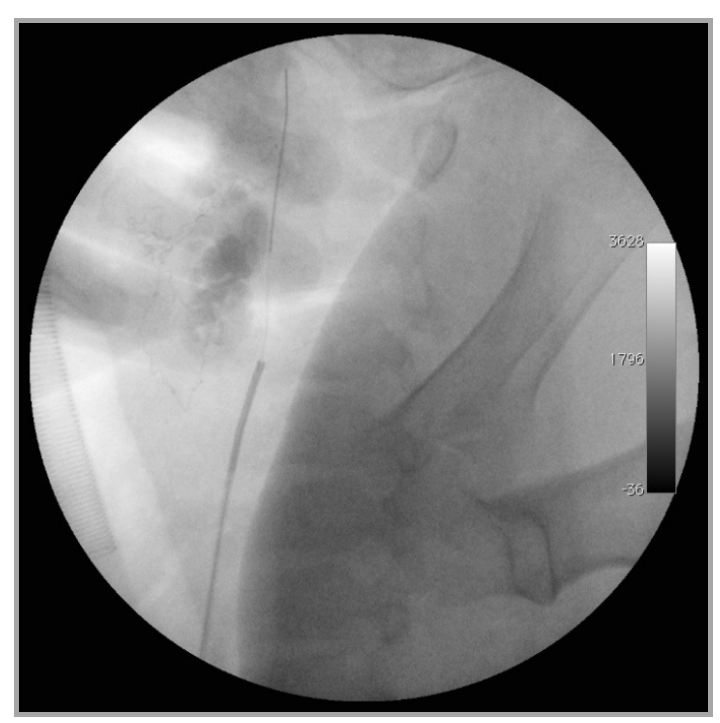

Figura 5. Imagem arteriográfica do momento em que o fio guia transpassa o local da lesão.

\subsection{AVALIAÇÃO DA VIABILIDADE DO PROCEDIMENTO}

Após a liberação do stent revestido (Figura 6), nova arteriografia foi realizada para que se evidenciasse tanto o posicionamento do stent, quanto a presença de fluxo sanguíneo adequado no local da implantação e na extremidade distal (Figura 7).

O fluxo sanguíneo local e distal à lesão foi documentado por ultrassonografia Doppler (Sonosite Titan $^{\circledR}$ ) após a indução anestésica, antes do início do procedimento e imediatamente após a realização do procedimento endovascular. Foram avaliados o diâmetro arterial máximo (em centímetros), a velocidade de pico sistólico (em centímetros por segundo), a velocidade de pico diastólico (em centímetros por segundo) e o índice de resistência. Estas medidas foram realizadas com o intuito de caracterizar alterações nas características do fluxo sanguíneo após a implantação do stent.

Foi considerado sucesso do procedimento, a caracterização nos exames após o tratamento endovascular, a localização adequada do stent, com fluxo de contraste em 
seu interior e sem a presença de extravasamento de contraste.

Após a realização da ultrassonografia doppler, ainda sob anestesia, os animais receberam dose letal de cloreto de potássio. Os animais foram submetidos a necrópsia para observação do local da lesão e dos tecidos adjacentes.
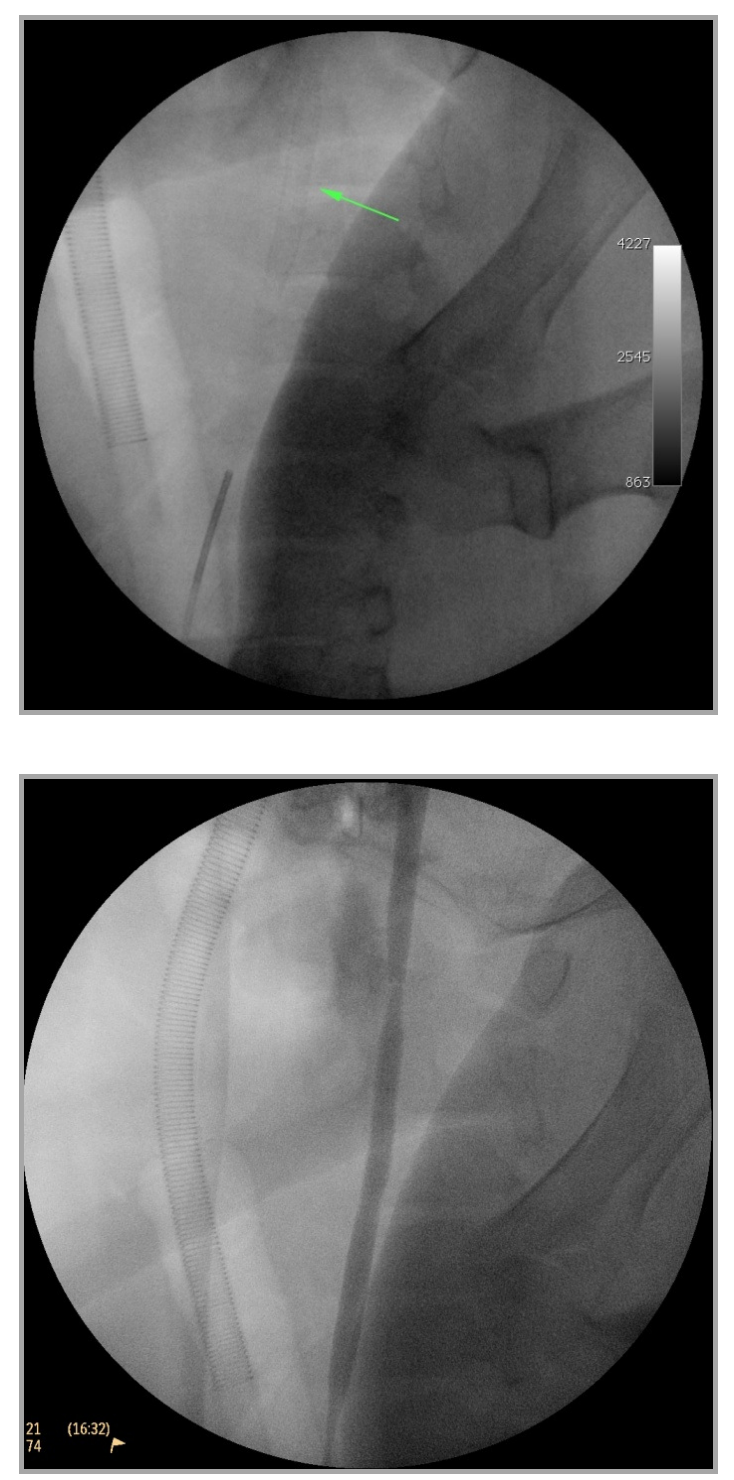

Figura 6. Posicionamento adequado do stent revestido liberado com sucesso.

Figura 7. Perviedade arterial e bom fluxo sanguineo evidanciados em imagem arteriográfica de controle. 


\subsection{ANÁLISE ESTATÍSTICA}

Estatísticas paramétrica (Teste $t$ de Student) e não paramétrica (Teste de Postos e Sinais de Wilcoxon) foram aplicadas aos dados registrados, para comparar os parâmetros quantitativos de cada grupo antes e depois dos procedimentos. Para a comparação dos dados entre os quatro grupos, foi aplicado o Teste de Kruskal-Wallis. O teste "U” de Mann-Whitney foi utilizado na comparação de variáveia quantitativas entre o grupo controle e os demais agrupados. Foi adotado nível de probabilidade de $95 \%(\mathrm{p}<0,05)$. 


\section{4. $\quad$ Resultados}

O tempo total de intervenção operatória oscilou entre 38 e 118 minutos (média de 56,3 $\pm 19,1$ minutos). Após a produção do trauma controlado, o tempo restante do procedimento variou de 19 a 45 minutos (média de 27,4 $\pm 6,6$ minutos). O tempo operatório total e o tempo operatório pós-trauma controlado para cada animal e a média de cada grupo se encontram nas Tabelas 2 e 3, respectivamente. Não houve diferença estatística significante entre os grupos no tempo total da intervenção $(\mathrm{p}=$ 0,4408) nem no tempo pós-trauma controlado $(\mathrm{p}=0,3868)$. Entretanto, vale destacar que houve diminuição do tempo de execução do procedimento a medida que aumentou a experiência com o número de experimentos realizados. No grupo G4, encerrou-se o procedimento, caracterizando insucesso, quando em 20 minutos não se conseguiu cateterizar o coto distal.

Tabela 2. Valores relativos ao tempo operatório total (em minutos) utilizado em cada porco e a média do grupo-controle e dos grupos experimentais.

\begin{tabular}{|c|c|c|c|c|}
\hline \multirow[b]{2}{*}{ Porcos } & \multicolumn{4}{|c|}{ Grupos } \\
\hline & $\begin{array}{c}\text { G1 } \\
\text { (controle) }\end{array}$ & $\begin{array}{c}\text { G2 } \\
(33 \%)\end{array}$ & $\begin{array}{c}\text { G3 } \\
(66 \%)\end{array}$ & $\begin{array}{c}\text { G4 } \\
(100 \%)\end{array}$ \\
\hline 1 & 93 & 118 & 64 & 52 \\
\hline 2 & 52 & 65 & 53 & 59 \\
\hline 3 & 56 & 40 & 49 & 59 \\
\hline 4 & 47 & 48 & 41 & 61 \\
\hline 5 & 41 & 47 & 38 & 42 \\
\hline Média \pm desvio-padrão & $57,8 \pm 20,5$ & $63,6 \pm 31,8$ & $49,0 \pm 10,3$ & $54,6 \pm 7,8$ \\
\hline
\end{tabular}


Tabela 3. Valores relativos ao tempo operatório pós-trauma controlado (em minutos) utilizado em cada porco e à média do grupo-controle e dos grupos experimentais.

\begin{tabular}{lcccc}
\hline \multirow{2}{*}{ Porcos } & \multicolumn{4}{c}{ Grupos } \\
\cline { 2 - 5 } & $\begin{array}{c}\text { G1 } \\
\text { (controle) }\end{array}$ & $\begin{array}{c}\text { G2 } \\
\mathbf{( 3 3 \% )}\end{array}$ & $\begin{array}{c}\text { G3 } \\
\mathbf{( 6 6 \% )}\end{array}$ & $\begin{array}{c}\text { G4 } \\
(\mathbf{1 0 0 \%})\end{array}$ \\
\hline 1 & 38 & 45 & 29 & 33 \\
2 & 23 & 36 & 23 & 29 \\
3 & 29 & 21 & 20 & 30 \\
4 & 27 & 27 & 21 & 25 \\
5 & 22 & 25 & 19 & 26 \\
\hline Média \pm desvio-padrão & $27,8 \pm 6,4$ & $30,8 \pm 9,6$ & $22,4 \pm 4,0$ & $28,6 \pm 3,2$ \\
\hline $\mathrm{p}=0,386$ & & & &
\end{tabular}

Houve sucesso no procedimento endovascular, em todos os cinco porcos do G1 (porcos sem trauma provocado), em todos os cinco porcos do G2 (lesões com extensão circunferencial de 33\%), em quatro dos cinco porcos do G3 (lesões com extensão circunferencial de 66\%), e em um dos cinco porcos com transecção completa (Tabela 4) (Figura 8). Em um dos animais do grupo G3, foi evidenciado trombose no interior do stent imediatamente após a liberação deste. Em quatro dos cinco porcos do grupo G4, não foi possível transpassar o sítio da lesão com o fio guia.

Dados relativos às funções vitais monitoradas ao longo de todo o procedimento cirúrgico se encontram na Tabela 5.

Tabela 4. Evidências das imagens arteriográficas antes e depois da liberação de stent revestido em trauma controlado de artéria carótida comum esquerda.

\begin{tabular}{|c|c|c|c|c|c|c|c|c|c|c|c|c|c|c|c|c|c|c|c|c|}
\hline \multirow{2}{*}{$\begin{array}{l}\text { Achados arteriográficos } \\
\text { Pré-procedimento }\end{array}$} & \multicolumn{5}{|c|}{ G1 } & \multicolumn{5}{|c|}{ G2 } & \multicolumn{5}{|c|}{ G3 } & \multicolumn{5}{|c|}{ G4 } \\
\hline & 1 & 2 & 3 & 4 & 5 & 1 & 2 & 3 & 4 & 5 & 1 & 2 & 3 & 4 & 5 & 1 & 2 & 3 & 4 & 5 \\
\hline \multicolumn{21}{|l|}{$\begin{array}{l}\text { Boa contrastação e velocidade } \\
\text { de escoamento }\end{array}$} \\
\hline \multirow{2}{*}{\multicolumn{21}{|c|}{$\begin{array}{l}\text { Espasmo intenso, } \\
\text { extravasamento do contraste }\end{array}$}} \\
\hline & & & & & & & & & & & & & & & & & & & & \\
\hline \multirow{2}{*}{\multicolumn{21}{|c|}{$\begin{array}{l}\text { Espasmo intenso, } \\
\text { extravasamento do contraste }\end{array}$}} \\
\hline & & & & & & & & & & & & & & & & & & & & \\
\hline \multicolumn{21}{|l|}{ Extravasamento de contraste } \\
\hline \multirow{2}{*}{\multicolumn{21}{|c|}{$\begin{array}{l}\text { Espasmo intenso, } \\
\text { extravasamento do contraste, } \\
\text { sem contraste do coto distal }\end{array}$}} \\
\hline & & & & & & & & & & & & & & & & & & & & \\
\hline \multirow{2}{*}{$\begin{array}{l}\text { Extravasamento do contraste, } \\
\text { sem contraste do coto distal }\end{array}$} & & & & & & & & & & & & & & & & & & & & \\
\hline & & & & & & & & & & & & & & & & & & & & \\
\hline
\end{tabular}




\begin{tabular}{|c|c|c|c|c|c|c|c|c|c|c|c|c|c|c|c|c|c|c|c|c|}
\hline \multirow{2}{*}{$\begin{array}{l}\text { Achados arteriográficos } \\
\text { Pós-procedimento }\end{array}$} & \multicolumn{5}{|c|}{ G1 } & \multicolumn{5}{|c|}{ G2 } & \multicolumn{5}{|c|}{ G3 } & \multicolumn{5}{|c|}{ G4 } \\
\hline & 1 & 2 & 3 & 4 & 5 & 1 & 2 & 3 & 4 & 5 & 1 & 2 & 3 & 4 & 5 & 1 & 2 & 3 & 4 & 5 \\
\hline Boa contrastação e velocidad & & & & & & & & & & & & & & & & & & & & \\
\hline de escoamento & & & & & & & & & & & & & & & & & & & & \\
\hline $\begin{array}{l}\text { Boa contrastação } \\
\text { espasmo moderado e }\end{array}$ & & & & & & & & & & & & & & & & & & & & \\
\hline escoamento lentificado & & & & & & & & & & & & & & & & & & & & \\
\hline Sem contrastação, sem & & & & & & & & & & & & & & & & & & & & \\
\hline Escoamento, trombose local & & & & & & & & & & & & & & & & & & & & \\
\hline Inviável & & & & & & & & & & & & & & & & & & & & \\
\hline
\end{tabular}
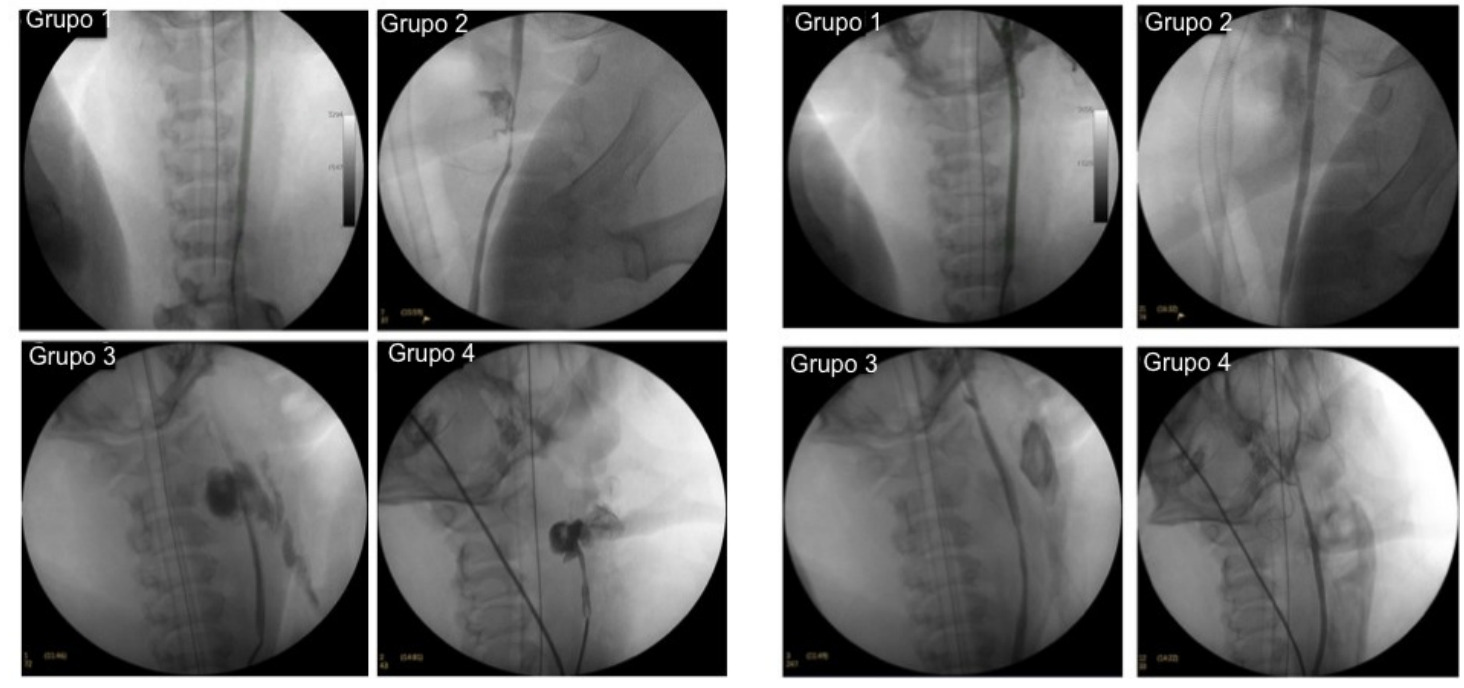

Figura 8. Imagens arteriográficas pré e pós-tratamento endovascular evidenciando procedimentos com sucesso nos quatro grupos.

Tabela 5. Valores médios e desvios-padrão dos parâmetros vitais monitorados ao longo do estudo.

\begin{tabular}{|c|c|c|c|c|c|c|c|c|}
\hline \multirow{2}{*}{ Parâmetros } & & \multicolumn{4}{|c|}{ Grupos } & \multicolumn{3}{|c|}{ Geral } \\
\hline & & $\begin{array}{c}\text { G1 } \\
\text { (controle) }\end{array}$ & $\begin{array}{c}\text { G2 } \\
(33 \%) \\
\end{array}$ & $\begin{array}{c}\mathbf{G 3} \\
(66 \%) \\
\end{array}$ & $\begin{array}{c}\text { G4 } \\
(100 \%) \\
\end{array}$ & Média & Mín. & Máx. \\
\hline $\begin{array}{l}\text { Frequência } \\
\text { mínima }\end{array}$ & cardíaca & $88,4 \pm 11,9$ & $87,4 \pm 8,9$ & $74,0 \pm 5,1$ & $75,6 \pm 11,3$ & $81,4 \pm 11,2$ & 60 & 109 \\
\hline $\begin{array}{l}\text { Frequência } \\
\text { máxima }\end{array}$ & cardíaca & $131,8 \pm 9,8$ & $133,2 \pm 9,3$ & $119,6 \pm 9,5$ & $126,2 \pm 12,8$ & $127,7 \pm 11,1$ & 108 & 145 \\
\hline \multicolumn{2}{|c|}{ Pressão arterial mínima } & $50,6 \pm 4,5$ & $49,6 \pm 9,7$ & $40,2 \pm 8,0$ & $45,6 \pm 6,3$ & $44,0 \pm 9,4$ & 28 & 59 \\
\hline \multicolumn{2}{|c|}{ Pressão arterial máxima } & $100,4 \pm 8,1$ & $129,6 \pm 7,4$ & $111,2 \pm 6,8$ & $112,4 \pm 12,8$ & $113,4 \pm 13,6$ & 93 & 140 \\
\hline \multicolumn{2}{|c|}{ PVC mínima } & $5,8 \pm 0,8$ & $7,0 \pm 1,4$ & $6,2 \pm 1,3$ & $5,4 \pm 1,1$ & $6,1 \pm 1,3$ & 4 & 8 \\
\hline \multicolumn{2}{|l|}{ PVC máxima } & $11,4 \pm 1,8$ & $11,0 \pm 1,6$ & $13,0 \pm 1,6$ & $11,2 \pm 1,6$ & $11,6 \pm 1,7$ & 9 & 15 \\
\hline
\end{tabular}

Os parâmetros avaliados por ultrassonografia Doppler em 15 procedimentos 
$(70,0 \%)$ bem sucedidos se encontram na Tabela 6.

Tabela 6. Parâmetros ultrassonográficos em 15 procedimentos endovasculares bem sucedidos após produção de trauma perfurante controlado.

\begin{tabular}{|c|c|c|c|c|c|c|c|c|c|}
\hline \multirow{3}{*}{\multicolumn{2}{|c|}{ Grupos }} & \multicolumn{8}{|c|}{ Parâmetros ultrassonográficos } \\
\hline & & \multicolumn{2}{|c|}{$\begin{array}{l}\text { Diâmetro arterial } \\
\text { máximo }(\mathrm{cm})\end{array}$} & \multicolumn{2}{|c|}{$\begin{array}{l}\text { Velocidade de pico } \\
\text { sistólico }(\mathrm{cm} / \mathrm{s})\end{array}$} & \multicolumn{2}{|c|}{$\begin{array}{l}\text { Velocidade de pico } \\
\text { diastólico }(\mathrm{cm} / \mathrm{s})\end{array}$} & \multicolumn{2}{|c|}{ Índice de resistência } \\
\hline & & Antes & Depois & Antes & Depois & Antes & Depois & Antes & Depois \\
\hline \multirow{5}{*}{ G1 } & 1 & 0,49 & 0,37 & 44,6 & 69,8 & 13,6 & 17,4 & 0,70 & 0,75 \\
\hline & 2 & 0,49 & 0,38 & 41,7 & 83,4 & 18,4 & 24,2 & 0,58 & 0,71 \\
\hline & 3 & 0,56 & 0,53 & 59,0 & 49,4 & 19,4 & 24,2 & 0,67 & 0,61 \\
\hline & 4 & 0,51 & 0,47 & 52,0 & 58,0 & 18,3 & 22,5 & 0,61 & 0,63 \\
\hline & 5 & 0,48 & 0,48 & 44,6 & 47,6 & 12,1 & 16,6 & 0,73 & 0,79 \\
\hline \multirow{5}{*}{ G2 } & 1 & 0,63 & 0,37 & 34,9 & 49,4 & 11,6 & 23,1 & 0,67 & 0,53 \\
\hline & 2 & 0,47 & 0,41 & 68,8 & 84,0 & 18,4 & 22,0 & 0,73 & 0,73 \\
\hline & 3 & 0,54 & 0,54 & 89,2 & 100,8 & 14,5 & 29,0 & 0,84 & 0,70 \\
\hline & 4 & 0,44 & 0,44 & 65,9 & 67,7 & 28,0 & 30,0 & 0,90 & 0,56 \\
\hline & 5 & 0,53 & 0,57 & 52,3 & 54,0 & 8,7 & 26,4 & 0,83 & 0,62 \\
\hline \multirow{4}{*}{ G3 } & 2 & 0,47 & 0,52 & 58,2 & 37,6 & 15,5 & 9,8 & 0,73 & 0,85 \\
\hline & 3 & 0,44 & 0,41 & 66,9 & 70,8 & 6,8 & 10,7 & 0,76 & 0,85 \\
\hline & 4 & 0,50 & 0,51 & 49,4 & 62,0 & 22,3 & 46,9 & 0,75 & 0,58 \\
\hline & 5 & 0,52 & 0,45 & 59,0 & 79,5 & 18,4 & 38,8 & 0,69 & 0,51 \\
\hline G4 & 2 & 0,47 & 0,30 & 64,0 & 74,6 & 16,0 & 27,1 & 0,73 & 0,64 \\
\hline
\end{tabular}

A análise dos valores médios desses parâmetros ultrassonográficos nos diferentes grupos para 15 procedimentos bem sucedidos revelou que houve redução significativa no diâmetro arterial máximo após o procedimento (de 0,50 $\pm 0,04 \mathrm{~mm}$ para $0,45 \pm 0,07 \mathrm{~mm} ; t=0,02 ; \mathrm{T}=11,5 ; \mathrm{p}<0,025)$. As velocidades de pico sistólico e, especialmente, diastólico aumentaram significativamente após o procedimento, mas não houve diferenças nos valores médios do índice de resistência observados antes e depois do procedimento (Tabela 7).

Em relação aos outros parâmetros, na comparação entre os grupos, não houve diferenças estatisticamente relevantes (Tabela 8). Excluimos nesta análise o grupo 4, pois neste, uma vez que houve apenas um procedimento com sucesso, impossibilitou sua análise comparativa com os demais grupos 
Tabela 7. Parâmetros ultrassonográficos (média \pm desvio-padrão) avaliados antes e após a colocação de stents revestidos na artéria carótida comum esquerda após a produção de trauma penetrante controlado no conjunto de 14 procedimentos bem sucedidos.

\begin{tabular}{lccc}
\hline Parâmetros ultrassonográficos & Antes & Depois & $\begin{array}{l}\text { Teste de postos e } \\
\text { sinais de Wilcoxon }\end{array}$ \\
\hline $\begin{array}{l}\text { Diâmetro arterial máximo (cm) } \\
\begin{array}{l}\text { Velocidade de pico sistólico } \\
(\mathrm{cm} / \mathrm{s})\end{array}\end{array}$ & $0,50 \pm 0,04$ & $0,45 \pm 0,07$ & $\mathrm{~T}=15 ; \mathrm{p}<0.025$ \\
$\begin{array}{l}\text { Velocidade de pico diastólico } \\
(\mathrm{cm} / \mathrm{s})\end{array}$ & $16.14 \pm 5.53$ & $24.40 \pm 9.96$ & $\mathrm{~T}=9 ; \mathrm{p}<0.005$ \\
\begin{tabular}{l} 
Indice de resistência \\
\hline
\end{tabular} & $0.73 \pm 0.08$ & $0.67 \pm 0.11$ & $\mathrm{~T}=24.5 ; \mathrm{p}>0.05$ \\
\hline
\end{tabular}

Tabela 8. Parâmetros ultrassonográficos (média \pm desvio-padrão) avaliados antes e após a colocação de stents revestidos na artéria carótida comum esquerda após a produção de trauma perfurante controlado no grupo-controle e nos grupos experimentais.

\begin{tabular}{lcccc}
\hline $\begin{array}{l}\text { Parâmetros } \\
\text { ultrassonográficos }\end{array}$ & G1 & G2 & G3 & $\begin{array}{c}\text { Teste de } \\
\text { Kruskal- } \\
\text { Wallis }\end{array}$ \\
\hline
\end{tabular}

Diâmetro arterial máximo

(cm)

Antes

Depois

Diferença média

$\begin{array}{cccc}0,50 \pm 0,03 & 0,52 \pm 0,07 & 0,47 \pm 0,04 & \\ 0,44 \pm 0,06 & 0,46 \pm 0,08 & 0,47 \pm 0,05 & \mathrm{H}=1,75 \\ -0,06 & -0,06 & -0,01 & \mathrm{p}>0,10\end{array}$

Velocidade de pico sistólico

$(\mathrm{cm} / \mathrm{s})$

Antes

Depois

Diferença média

$\begin{array}{cccc}48,48 \pm 7,05 & 62,22 \pm 20,18 & 54,84 \pm 10,04 & \\ 61,64 \pm 15,00 & 71,18 \pm 21,35 & 62,47 \pm 18,05 & \mathrm{H}=0,22 \\ 13.26 & 8.96 & 4.10 & \mathrm{p}>0,30\end{array}$

Velocidade de pico diastólico

$(\mathrm{cm} / \mathrm{s})$

Antes

Depois

Diferença média

$\begin{array}{cccc}16,36 \pm 3,28 & 16,24 \pm 7,48 & 15,75 \pm 6,59 & \\ 20,98 \pm 3,70 & 26,10 \pm 3,51 & 23,26 \pm 19,11 & H=0,61 \\ 4,62 & 6,66 & 10,8 & p>0,30\end{array}$

Índice de resistência
Antes
$0,66 \pm 0,06$
$0,79 \pm 0,09$
$0,73 \pm 0,03$
Depois
$0,70 \pm 0,07$
$0,62 \pm 0,08$
$0,69 \pm 0,17$
$\mathrm{H}=4,52$;
Diferença média
$0,04-0,16$
$-0,03$
$\mathrm{p}>0,08$

Entretanto, vale destacar que, quando comparamos isoladamente o índice de resistência do grupo controle G1 (n=5), sem lesão arterial, com os demais grupos, com lesão arterial G2, G3 e G4 (n=10), evidenciamos aumento do índice de resis- 
tência naquele (em média $0,04 \pm 0,068$ ) e diminuição do índice de resistência nestes (em média $0,1 \pm 0,019)$. Tal variação é marginalmente significativa $(\mathrm{P}=0,05)$.

Em relação aos achados da necróspia destes animais, foi notado presença constante de extenso hematoma nos animais submetidos a lesão vascular, independentemente da extensão circunferencial desta. Importante destacar a peça da necrópsia do animal do procedimento bem sucedido do Grupo 4, onde foi visibilizado os dois cotos do vaso lesado conectados pelo stent revestido (Figura 9).

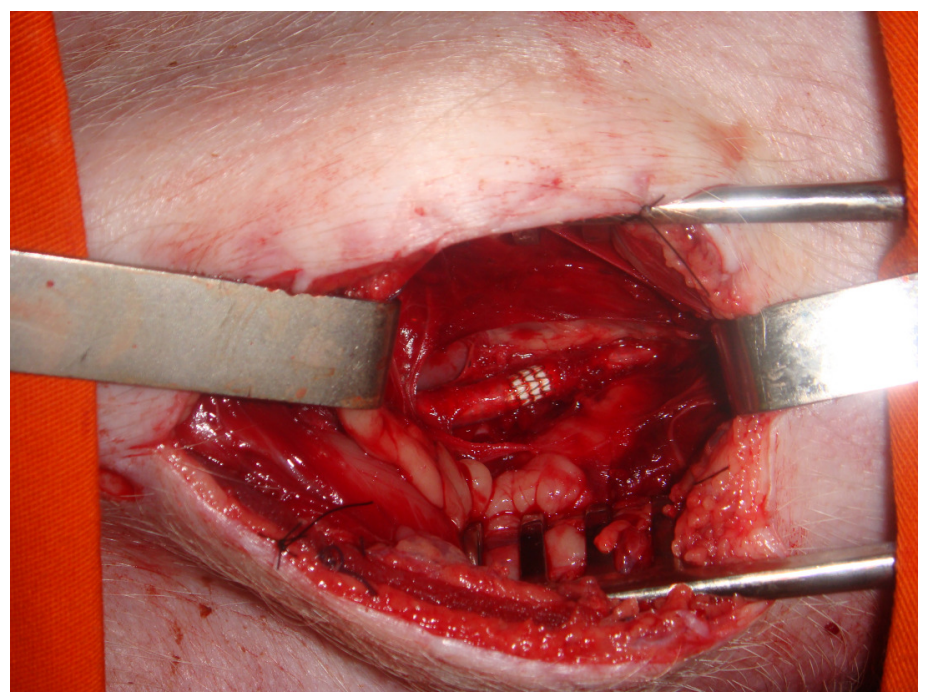

Figura 9. Imagem de necrópsia evidenciando os cotos arteriais conectados pelo stent revestido. 


\section{Discussão}

Em situações clínicas de trauma arterial periférico, como em casos de hematomas extenso e dificuldade de acesso, o tratamento convencional, via cirurgia aberta, é controverso.

Neste contexto, o uso de stents revestidos vem sendo recomendado para o reparo de lesões traumáticas da artéria femoral ${ }^{13,26}$, das artérias subclávia e axilar ${ }^{15,26}$, uma vez que promovem a redução das altas taxas de morbidade e mortalidade associadas com a exploração cirúrgica aberta.

Ainda não há consenso no que tange ao tratamento de lesões das artérias carótida comum e interna de pacientes após traumas penetrantes e contusos. Com base nos achados do exame clínico, isolados ou associados com imagens radiológicas, indicase ainda, predominantemente, a exploração aberta de urgência ou eletiva, apesar de em alguns casos se evidenciou a viabilidade e a segurança do tratamento endovascu$\operatorname{lar}^{11,21,22,24}$.

As vantagens da utilização de stents incluem, sobretudo, a sua introdução minimamente invasiva e o seu posicionamento através de um território remoto mediante acesso percutâneo ${ }^{5}$. Todavia, eles têm sido extensivamente estudado e indicados predominantemente em artérias doentes, com aterosclerose, aneurismas e dissecções, já que a grande maioria dos estudos realizados se refere justamente a essas condições vasculares ${ }^{26}$. 
Desta forma, o reparo endovascular de lesões em pacientes saudáveis deve ser melhor avaliado, de modo a estabelecer a viabilidade do uso de stents revestidos no tratamento de traumas contusos ou perfurantes, assim como para produzir evidências sobre o comportamento a longo prazo desses materiais. Além disso, deve-se identificar as características das lesões que as torne passíveis de tratamento pela abordagem endovascular.

As questões que se colocam referente a qual material mais adequado, qual o comportamento do vaso tratado a curto e longo prazo, qual o comportamento do material em áreas de bifurcações e sujeitas a flexões, limitam o uso desta terapêutica. Evidentemente, repostas a essas questões só podem ser encontradas, inicialmente, por meio de estudos experimentais para posterior disseminação para o uso clínicoprático.

Nosso objetivo primário neste estudo foi o desenvolvimento de um modelo animal em que se pudesse avaliar o reparo endovascular de traumas penetrantes nas artérias periféricas.

Houve sucesso no posicionamento dos stents revestidos em $100 \%$ dos animais do grupo-controle (sem trauma carotídeo) e em 100\% dos animais cujos traumas controlados possuíam extensão circunferencial de 33\% (<50\%). O grupo 1 permitiu a equipe, após os casos do projeto piloto, melhor capacitação no procedimento. Este fato explica porque nos grupos 3 e 4 o procedimento durou menos tempo, apesar de mais complexos devido as lesões. O estudo demonstra claramente que este modelo pode também ser aplicado para treinamento de equipes na técnica endovascular com melhora do desempenho em uma situação experimental antes da prática clínica.

Sucesso foi observado em quatro $(80,0 \%)$ animais com traumas abrangendo ex- 
tensão circunferencial de $66 \%$ ( $>50 \%)$. O procedimento mostrou-se viável também em um dos cinco animais em que se realizou secção completa (100\%). Portanto, pudemos observar a viabilidade do procedimento experimental, bem como associar o sucesso do procedimento com a extensão circunferencial da lesão traumática. Não encontramos dados na literatura que tenham avaliado experimentalmente a associação entre a extensão circunferencial da lesão traumática e a viabilidade de terapêutica endovascular.

Os porcos são bons modelos experimentais para o estudo do sistema cardiovascular, uma vez que possuem características importantes similares às do ser humano no que concerne à anatomia e à fisiologia cardiovascular ${ }^{62}$. Por outro lado, existem diferenças entre esses animais e o homem com relação à anatomia cerebrovascular extracraniana (Figura 8), razão pela qual se deve proceder a considerações cuidadosas antes de se aplicarem os achados preliminares deste estudo à prática cirúrgica.

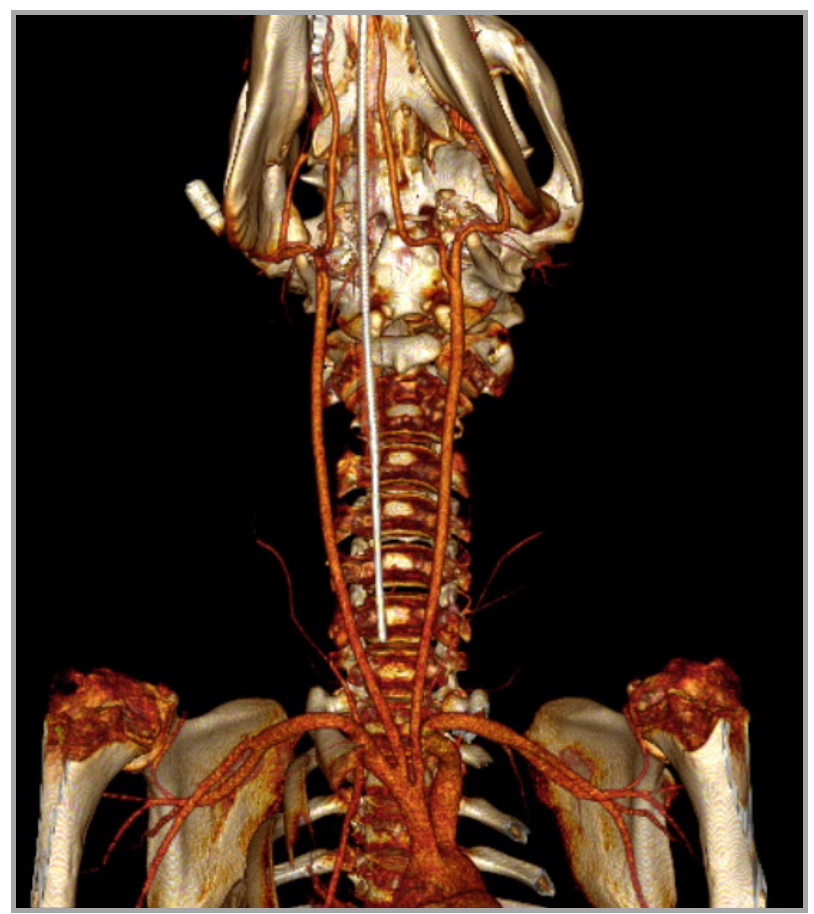

Figura 10. Anatomia vascular suína evidenciada em reconstrução de angiotomografia computadorizada. 
Em relação a análise dos parâmetros ultrassonográficos, não foi evidenciada diferença significativa nos valores do índice de resistência registrados antes e depois do procedimento, nem na comparação entre os grupos G1, G2 e G3 isolados. Entretanto, vale ressaltar que, se comparado o grupo sem lesão com os demais grupos, houve aumento do índice de resistência no grupo-controle (sem lesões), enquanto esse índice diminuiu nos grupos com lesões. Esta diferença no índice entre o grupo com lesão em relação ao grupo sem lesão ocorreu supostamente em decorrência da vasorreatividade cerebral. Nos animais com lesão, uma vez que há insuficiência vascular cerebral, ocorre vasodilatação reacional, o que acarreta em diminuição da resistência vascular ao fluxo sanguíneo, o que não acontece no grupo controle (sem lesão). ${ }^{68}$

O diâmetro arterial máximo, local de maior diâmetro aferível na carótida, reduziu significativamente em todos os três grupos na avaliação pós-procedimento, sem diferenças entre os grupos. Explica-se este fenômeno pelo importante grau de espasmo vascular que deve ser esperado naqueles que conduzirem estudos com a técnica endovascular em $\operatorname{porcos}^{62}$. Os valores relativos à velocidade de pico sistólico e diastólico, por sua vez, aumentaram significativamente após o procedimento, e novamente não se observou diferenças entre os grupos. O aumento da velocidade é um aspecto esperado em decorrência da diminuição da complacência do vaso no local de implantação do $s t e n t{ }^{66}$. Já é conhecido na prática clínica através do seguimento de pacientes com stents não revestidos na carótida interna colocados para tratamento de estenoses ateroscleróticas. Este fato também presente neste estudo indica que a veloci- 
dade espectral com o mapeamento duplex pode ser utilizada para seguimento clínico com a ressalva que os padrões de velocidade mudam com a inserção de stents revestidos. ${ }^{67}$

Houve dificuldades para a avaliação ultrassonográfica após o procedimento. A abordagem cirúrgica aberta realizada para a secção da artéria carótida acarreta presença de ar atmosférico entre as camadas, o que, somado ao hematoma subsequente e às limitações do ultrassom para a observação de segmentos mais distais da artéria carótida, leva à perda da acurácia do controle ecográfico pós-trauma no modelo descrito.

A análise desses parâmetros ultrassonográficos não incluiu medidas observadas no vaso distais ao posicionamento do stent revestido, exceto por três animais do grupo-controle, um animal com lesão $<50 \%$ e um animal com lesão $>50 \%$ em que foi possível a visualização deste segmento. Nesses animais, as medidas observadas antes e depois do posicionamento do stent foram bastante similares.

Houve falhas no desenho do estudo. A realização sequencial, e não de forma aleatória dos grupos, criou um viés na aferição de tempo do procedimento, uma vez que houve aumento de experiência na realização do procedimento a medida que se aumentou o número de procedimento realizados. Importante destacar também, a discrepância entre os achados arteriográficos e ultrassonográficos. Houveram animais em que a velocidade de escoamento do contraste observada na arteriografia divergiu com a velocidade de pico sistólico encontrada na ultrassonografia Doppler. Este fato se deve supostamente pela subjetividade inerente a interpretação dos dados arteriográficos e a alteração no grau de espasmo arterial entre a realização da arteriografia e da ultrassonografia Doppler. 
Com o estudo realizado, foi criado um modelo experimental inédito que servirá para o suporte de outros estudos experimentais com a utilização de animais e técnicas endovasculares. Os resultados deste estudo ajudam efetivamente a entender e suportar o uso de técnica endovascular no tratamento de vítimas de lesões vasculares periféricas causadas por trauma penetrante. Este modelo ajuda a analisar os tipos de stents que podem ser utilizados e as conseqüências do seu implante, além de permitir o treinamento do implante destes materiais. 


\section{Conclusões}

O modelo experimental proposto para avaliar a viabilidade do reparo endovascular com uso de stents revestidos em lesões vasculares periféricas decorrentes de traumas penetrantes mostrou ser factível.

O sucesso do reparo endovascular com uso de stents revestidos foi associado de forma inversamente proporcional com a extensão circunferencial da lesão perfurante provocada na parede arterial. 


\section{Anexos}

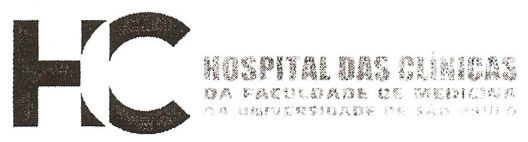

\section{APROVAÇÃO}

A Comissão de Ética para Análise de Projetos de Pesquisa CAPPesa da Diretoria Clínica do Hospital das Clínicas e da Faculdade de Medicina da Universidade de São Paulo, em sessão de 16/12/2009, AProVOU O Protocolo de Pesquisa n 0603/09, intitulado: 'TRATAMENTO ENDOVASCULAR DE TRAUMA ARTERIAL PERIFÉRICO COM USO DE STENTS REVESTIDOS. ESTUDO EXPERIMENTAL EM PORCOS" apresentado pelo Departamento de CIRURGIA.

Cabe ao pesquisador elaborar e apresentar à CAPPesq, os relatórios parciais e final sobre a pesquisa (Resolução do Conselho Nacional de Saúde n¹96, de 10/10/1996, inciso (X.2, letra "c").

Pesquisador (a) Responsável: Prof. Dr. Erasmo Simão da Silva

Pesquisador (a) Executante: Sergio Quilici Belczak

CAPPesq, 16 de Dezembro de 2009

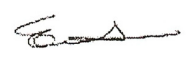

Prof. Dr. Eduardo Massad Presidente da Comissão de

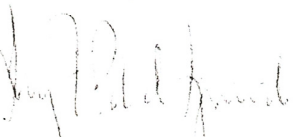

Ética para Análise de Projetos de Pesquisa

Prof. Dr. Luiz Francisen Poll de Figueiredo

Coordenador do Programa de fíus-ciraduaçăo Clínica Cirúrgica - FMUSP

Comissão de Ética para Análise de Projetos de Pesquisa do HCFMUSP e da FMUSP Diretoria Clínica do Hospital das Clínicas da Faculdade de Medicina da Universidade de São Paulo Rua Ovidio Pires de Campos, 225. $5^{\circ}$ andar - CEP 05403010 - Săo Paulo - SP Fone: 01130696442 Fax: 01130696492 e-mail: cappesq@hcnet.usp.br / secretariacappesq2@hcnet.usp.br 


\section{Referências}

1. Girolami A. Changes in the causes of trauma in the last 20 years. Trauma 1999; $1: 3-11$.

2. Compton C, Rhee R. Peripheral vascular trauma. Perspect Vasc Surg Endovasc Ther 2005;17:297-307.

3. du Toit DF, van Schalkwyk GD, Wadee SA, Warren BL. Neurologic outcome after penetrating extracranial arterial trauma. J Vasc Surg 2003;38:257-62.

4. Peck MA, Rasmussen TE. Management of blunt peripheral arterial injury. Perspect Vasc Surg Endovasc Ther 2006;18:159-173.

5. Katsanos K, Sabharwal T, Carrell T, Dourado R, Adam A. Peripheral endografts for the treatment of traumatic arterial injuries. Emerg Radiol 2009;16:175-184.

6. Ruppert V, Sadeghi-Azandaryani M, Mutschler W, Steckmeier B. Vascular injuries in extremities. Chirurg 2004;75:1229-1240.

7. Saad NE, Saad WE, Davies MG, Waldman DL, Fultz PJ, Rubens DJ. Pseudoaneurysms and the role of minimally invasive techniques in their management. Radiographics 2005;25(Suppl 1):173-189.

8. Demetriades D, Murray J, Sinz B, Myles D, Chan L, Sathyaragiswaran L, et al. Epidemiology of major trauma and trauma deaths in Los Angeles County. J Am Coll Surg 1998;187:373-383.

9. Yi AC, Palmer E, Luh GY, Jacobson JP, Smith DC. Endovascular treatment of carotid and vertebral pseudoaneurysms with covered stents. Am J Neuroradiol 2008; 29:983-7.

10. Maras D, Lioupis C, Magoufis G, Tsamopoulos N, Moulakakis K, Andrikopou- 
los V. Covered stent-graft treatment of traumatic internal carotid artery pseudoaneurysms: a review. Cardiovasc Intervent Radiol 2006;29:958-968.

11. Nicholson AA. Vascular radiology in trauma. Cardiovasc Intervent Radiol 2004;27:105-120.

12. White R, Krajcer Z, Johnson M, Williams D, Bacharach M, O'Malley E. Results of a multicenter trial for the treatment of traumatic vascular injury with a covered stent. J Trauma 2006;60:1189-1196.

13. Starnes BW, Arthurs ZM. Endovascular management of vascular trauma. Perspect Vasc Surg Endovasc Ther 2006;18(Suppl. 2):114-129.

14. Vinces FY, Sparling DC. Endovascular treatment of a combined pseudaneurysm and arteriovenous fistula of the subclavian artery caused by a gunshot wound to the chest. J Thor Cardiovasc Surg 2005; 130(1):225-7.

15. Xenos ES, Freeman M, Stevens S, Cassada D, Pacanowski J, Goldman M. Covered stents for injuries of subclavian and axillary arteries. J Vasc Surg 2003;38:451454.

16. Parodi JC, Scholnholz C, Ferreira LM, Bergan J. Endovascular stent-graft treatment of traumatic arterial lesions. Ann Vasc Surg. 1999;13(2):121-129.

17. Alimi YS, Hakam Z, Hartung O, Boufi M, Barthèlemy P, Aissi K, et al. Efficacy of Viabahn in the treatment of severe superficial femoral artery lesions: which factors influence long-term patency? Eur J Vasc Endovasc Surg 2008;35:346-352.

18. Spirito R, Trabattoni P, Pompilio G, Zoli S, Agrifoglio M, Biglioli P. Endovascular treatment of a post-traumatic tibial pseudoaneurysm and arteriousfistula: Case report na review of the literature. J Vasc Surg 2007; 45:1076-9.

19. Cohen J, Leker RR, Gotkine M, Gomoni M, Ben-Hur T. Emergent stenting to treat patients with carotid artery dissection. Stroke 2003; 34:254-7. 
20. Tseng A, Ramaiah V, Rodriguez-Lopez JA, Perkowishi PE, Del Santo PB, Gowda $\mathrm{RG}$ et al. Emergent endovascular treatment of a spontaneous internal carotid artery dissection with pseudoaneurysm. J Endovasc Ther 2003; 10:643-6.

21. Assadian A, Senekowitsch C, Rotter R, Zölss C, Strassegger J, Hagmüller GW. Long-term results of covered stent repair of internal carotid artery dissections. J Vasc Surg 2004;40:484-487.

22. Feugier P, Vulliez A, Bina N, Floccard B, Allaouchiche B. Urgent endovascular covered-stent treatment of internal carotid artery injury caused by a gunshot. Eur $\mathbf{J}$ Vasc Endovasc Surg 2007; 34(6):663-5.

23. Li W, D’Ayala M, Hirshberg A, Briggs W, Wise L, Tortolani A. Comparison of conservative and operative treatment for blunt carotid injuries: analysis of the $\mathrm{Na}-$ tional Trauma Data Bank. J Vasc Surg 2007; 51(3):593-9.

24. Du Bose J, Recinos G, Teixeira PG, Inaba K, Demetriades D. Endovascular stenting for the treatment of traumatic internal carotid injuries: expanding experience. J Trauma 2008;65(6):1561-6.

25. Moulakakis KG, Mylonas S, Avgerinos E, Kotsis T, Liapis CD. An update of the role of endovascular repair in blunt carotid artery trauma. Eur J Vasc Endovasc Surg $2010 ; 40: 312-9$.

26. França LHG, Pereira AH. Update on vascular endoprotheses (stents): from experimental studies to clinical practice. J Vasc Bras 2008; 7(4). Scielo doi: 10.159/ S167754492008000400010.

27. Lobato AC. Stents vasculares. In: Lobato AC. Cirurgia endovascular. São Paulo: Instituto de Cirurgia Vascular e Endovascular de São Paulo; 2006. p. 83-96.

28. Nelken N, Schneider PA. Advances in stent technology and drug-eluting stents. Surg Clin North Am 2004; 84:1203-36.

29. Leung DA, Spinosa DJ, Hagspiel KD, Angle JF, Matsumoto AH. Selection of stents for treating iliac arterial occlusive disease. 
30. Mangell P, Malina M, Vogt K. Are self-expansible stents superior to balloonexpanded in dilating aortas? An experimental study in pigs. Eur J Endovsc SUrg 1996; 12:287-94.

31. Andrews RT, Venbrux AC, Magee CA, Bova DA. Placement of a flexible endovascular stent across the femoral joint: an in vivo study in the swine model. J Vasc Interv Radiol 1999; 10:1219-28.

32. Henry M, Klonaris C, Amor M, Henry I, Tzvetanov K. State of the art: which stent for which lesion in peripheral interventions? Tex Heart Inst J 2000; 27:119-26.

33. Rolland PH, Mekkaoui C, Vidal V. Compliance matching stent placement in the carotid artery of the swine promotes optimal blood flow and attenuates restenosis. Eur J Vasc Endovasc Surg 2004; 28:431-8.

34. Jahnke T, Voshage G, Müller-Hülsbeck S, Grimm J, Heller M, Brossmann J. Endovascular placement of self-expanding nitinol coil stents for the treatment of femoro-popliteal obstructive disease. J Vasc Interv Radiol 2002; 13:257-66.

35. Bosiers M, Deloose K, Verbist J, Peeters P. Carotid artery stenting: which stent for which lesion? Vascular 2005; 13:205-10.

36. Faxon DP, Sanborn TA, Weber VJ. Restenosis following transluminal angioplasty in experimental atherosclerosis. Arteriosclerosis 1984; 4:189-95.

37. Richter GM, Palmaz JC, Noeldge G, Tio F. Relationship between blood flow, thrombus, and neointima in stents. J. Vasc Interv Radiol 1999; 10:598-604.

38. Palmaz JC. Intravascular stents: tissue interactions and design considerations. Am j Roentgenol 1993; 160:613-8.

39. Hamuro M, Palmaz JC, Sprague EA, Fuss C, Luo J. Influence of stent edge angle on endothelization in an in vitro model. J Vasc Interv Radiol 2001; 12:607-11.

40. Palmaz JC. New advances in endovascular technology. Tex Heart Inst J 1997; 24:156-9. 
41. Lammer J, Dake MD, Bleyn J. Peripheral arterial obstruction: prospective study of treatment with a transluminally placed self-expanding stent-graft. International Trial Study Group. Radiology 2000; 217:95-104.

42. Lammer J. Femoropopliteal artery obstruction: from de balloon to the stent graft. Cardiovasc Intervent Radiol 2001; 24:73-83.

43. Wiesinger B, Beregi JP, Oliva PL. PTFE-covered self-expanding nitinol stents for the treatment of severe iliac and femoral artery stenosis and occlusions: final results from a prospective study.

44. Grudtner MA, Pereira AH, Costa LFM. Efeitos a curto prazo de stents não recobertos e recobertos com politetrafluoretileno em aorta de suínos: um modelo experimental.

45. Ombrellaro MP, Stevens SL, Sciarrotta J, Schaeffer DO, Freeman MB, Goldman MH. Effect of balloon-expandable and self expanding stent fixation on endoluminal polytetrafluoroethylene graft healing. Am J Surg 1997; 173:461-6.

46. Cejna M, Virmani R, Jones R. Biocompatibility and performance of the wallstent and several covered stents in a sheep iliac artery model. J Vasc Interv Radiol 2001; 12:351-8.

47. Marty B, Leu AJ, Mucciolo A, von Segesser LK. Biologic fixation of polyester versus polyurethane covered stents in a porcine model. J Vasc Interv Radiol 2002; 13:601-7.

48. ANgelini GD, Lloyd C, Bush R, Johnson J, Newby AC. An external, oversized, porous polyester stent reduces vein graft neointima formation, cholesterol concentration, and vascular cell adhesion molecule 1 expression in cholesterol fed pigs. J Thorac Cardiovasc Surg 2002; 124:950-6.

49. Castañeda F, Ball SM, Wyffels PL, Young K, Li R. Assessment of a polyester covered nitinol stent in an atherosclerotic swine model. J Vasc Interv Radiol 2000; 11:483-91. 
50. Avino A, Johnson B, Bandyk D. Does prosthetic covering of nitinol stents alter healing characteristics or hemodynamics? J Endovasc Ther 2000; 7:469-78.

51. Dutra CF, Pereira AH. Digital morphometric analysis of the aortic wall in pigs following implantation of Dacron-covered stents versus non-covered stents. Acta Cir Bras 2004; 19:210-9.

52. Fontaine AB, Borsa JJ, Hoffer E, Bloch R, So C. Evaluation of silicone as an endovascular stent membrane: in vivo canine studies. Cardiovasc Interv Radiol 2001; 24:324-8.

53. Galloni M, Prunotto M, Santarelli A. Carbon coated stents implanted in porcine iliac an renal arteries: histologic and histomorphometric study. J Vasc Interv Radiol 2003; 14:1053-61.

54. Goggins WC, Fisher RA, Cohen DS, Tawes JW, Grimes MM. Effect of singleclose rapamycin-based immunosuppression on the development of cardiac allograft vasculopathy. J Heart Lung Transpl 1996; 15:790-5.

55. Moses JW, Leon MB, Popma JJ. Sirolimus-eluting stents versus standard stents in patients with stenosis in a antive coronary artery. N Engl J Med 2003; 349:131523.

56. Wong G, Li J, Hendricks G, Eslami MH, Rohrer MJ, Cutler BS. Inhibition of experimental neointimal hyperplasia by recombinant human thrombomodulin coated ePTFE stent grafts. J Vasc Surg 2008; 47:608-15.

57. Duda SH, Bosiers M, Lammer J. Drug-eluting bare nitinol stents for the treatment of atherosclerotic lesions in the superficial femoral artery: long term results from the SIROCCO trial. J Endovasc Ther 2006; 13:701-10.

58. Duda SH, Bosiers M, Lammer J. Sirolimus-aluting versus bare nitinol stent for obstructive superficial femoral artery disease: the SIROCCO II trial. J Vasc Interv Radiol 2005; 16:331-8. 
59. Fontaine AB, Borsa JJ, Dos Passos S. Evaluation of local abciximab delivery from the surface of a polymer-coated covered stent: in vivo canine studies. J Vasc Interv Radiol 2001; 12:487-92.

60. ANsel GM, Silver MJ, Botti CF Jr. Functional and clinical outcomes of nitinol stenting with and without abciximab for complex superficial femoral artery disease: a randomized trial. Catheter Cardiovasc Interv 2006; 67:288-97.

61. Tielliu IFJ, Verhoeven ELG, Zeebregts CJ, Prins TR, Span MM, van den Dungen JJAM. Endovascular treatment of popliteal artery aneurysms: Results of a prospective cohort study. J Vasc Surg 2005; 41:561-7.

62. Swindle MM. Swine in the laboratory: Surgery, anesthesia, imaging, and experimental techniques. 2nd ed. London/New York: CRC Press; 2007.

63. Lin PH, Chronos NA, Marijianowski MM. Carotid stenting using heparin-coated balloon-expandable stent reduces intimal hyperplasia in a balloon model. J Surg Res 2003; 112:84-90.

64. Lin PH, Chronos NA, Marijianowski MM. Heparin-coated balloon-expandable stent reduces intimal hyperplasia in the iliac artery in baboons. J Vasc Interv Radiol 2003; 14:603-11.

65. Nakayama Y, Nishi S, Ishibashi-Ueda H. Fabrication of drug eluting covered stents with micropores and differential coating of heparin and FK506. Cardiovasc Radiat Med 2003; 4:77-82.

66. Santiago C, Miller MT, Pigott JT, Whalen RC, Jones L, Camerota AJ. Duplex ultrasound velocity criteria for the stented carotid artery. J Vasc Surg 2008; 1:63-73.

67. Lal BK, Hobson II RW, Tofighi B, Kapadia I, Cuadra S, Jamil Z. Carotid artery velocity characteristics after carotid artery angioplasty and stenting. J Vasc Surg 2006; 3:523-26.

68. Chang TY, Liu HL, Lee TH, Kuan WC, Chang CH, Wu HC, Wu TC, Chang YJ. Change in cerebral perfusion after carotid angioplasty with stenting is related to cerebral vasoreactivity: a study using dynamic susceptibility-weighted contrast- 
enhanced MR imaging and functional MR imaging with a breath-holding paradigm. AJNR Am J Neuroradiol 2009; 7:1330-6. 